\title{
DYNAMICS ON TEICHMÜLLER SPACES AND SELF-COVERING OF RIEMANN SURFACES
}

\author{
EGE FUJIKAWA, KATSUHIKO MATSUZAKI, AND MASAHIKO TANIGUCHI
}

\begin{abstract}
A non-injective holomorphic self-cover of a Riemann surface induces a non-surjective holomorphic self-embedding of its Teichmüller space. We investigate the dynamics of such self-embeddings by applying our structure theorem of self-covering of Riemann surfaces and examine the distribution of its isometric vectors on the tangent bundle over the Teichmüller space. We also extend our observation to quasiregular self-covers of Riemann surfaces and give an answer to a certain problem on quasiconformal equivalence to a holomorphic self-cover.
\end{abstract}

\section{INTRODUCTION}

The Teichmüller space $T(R)$ of a hyperbolic Riemann surface $R$ is the quasiconformal deformation space of the complex structure of $R$. It can be considered as a complex Banach manifold, and actually it is biholomorphically equivalent to a bounded domain in a certain complex Banach space. When $R$ is of analytically finite type, $T(R)$ is a domain of holomorphy in $\mathbb{C}^{n}$. One of the central issues for such domains is to investigate the structure of the space of holomorphic self-maps and its certain subspaces.

For a complex Banach manifold $M$ in general, we denote by $\operatorname{End}(M)$ the semigroup of all holomorphic self-maps of $M$. It contains the distinguished subgroup $\operatorname{Aut}(M)$ consisting of all biholomorphic automorphisms of $M$. In the case where $M$ is the Teichmüller space $T(R)$, every element of $\operatorname{Aut}(T(R))$ is now known to be a geometric automorphism induced by a quasiconformal

2000 Mathematics Subject Classification. Primary 37F30; Secondary 30F60, 32G15.

E. Fujikawa

Department of Mathematics, Chiba University, 1-33 Yayoicho, Inage-ku, Chiba 263-8522, Japan. E-mail:fujikawa@math.s.chiba-u.ac.jp

K. Matsuzaki

Department of Mathematics, Okayama University, 3-1-1 Tsushima-Naka, Okayama 7008530, Japan. E-mail:matsuzak@math.okayama-u.ac.jp

M. Taniguchi

Department of Mathematics, Nara Women's University, Kitauoya-Nishimachi, Nara 6308506, Japan. E-mail:tanig@cc.nara-wu.ac.jp. 
automorphism of $R$, and hence $\operatorname{Aut}(T(R))$ can be identified with the quasiconformal mapping class group $\mathrm{MCG}(R)$ of the base Riemann surface $R$ (Earle and Gardiner [3], Markovic [12]). The key to understanding the structure of $\operatorname{End}(T(R))$ is such intertwinement between the geometric properties of $R$ and the analytic properties of $T(R)$, which allows us to treat it more concretely and obtain deeper results than those by the general theory. This is the reason why we do not discuss general domains in complex Banach spaces but restrict ourselves to Teichmüller spaces.

There are another kind of particular elements in $\operatorname{End}(T(R))$ raised analogously as in the classical Fatou-Bieberbach phenomena, i.e. injective, but not surjective, holomorphic self-maps in $\operatorname{End}\left(\mathbb{C}^{n}\right)$ for $n \geq 2$ (cf. [17]). However, for the bounded domain $T(R)$, it is easy to make an injective holomorphic selfmap in such a way that the closure of its image is properly contained in $T(R)$. To avoid these self-maps, we impose such a condition on $F \in \operatorname{End}(T(R))$ that $F$ preserves the infinity, namely, $F(p)$ tends to the boundary of $T(R)$ if and only if $p$ tends to the boundary. Remark that, for a bounded domain $M$ in $\mathbb{C}^{n}$, this condition is nothing but $F \in \operatorname{End}(M)$ is proper. Hence the existence of injective, but non-surjective, self-maps preserving the infinity forces $T(R)$ to be infinite dimensional.

Let $\operatorname{Emb}(T(R))$ denote the semigroup consisting of all injective holomorphic self-maps of $T(R)$ preserving the infinity, which is possibly larger than $\operatorname{Aut}(T(R))$. We call an element in $\operatorname{Emb}(T(R))$ a holomorphic self-embedding of $T(R)$. For the sake of simplicity, we consider only the elements of $\operatorname{End}(T(R))$ fixing the base point of $T(R)$ for the moment. We express this restriction by adding a subscript 0 , or more explicitly, by $\operatorname{End}_{0}(T(R)), \operatorname{Aut}_{0}(T(R))$ and $\operatorname{Emb}_{0}(T(R))$. Every element of $\operatorname{Aut}_{0}(T(R))$ is induced geometrically by an element of $\operatorname{Conf}(R)$, the group of all conformal automorphisms of $R$. Similar to this case, typical examples of elements in $\operatorname{Emb}_{0}(T(R))$ come from a semigroup $\operatorname{Cov}(R)$, which consists of all holomorphic self-covers of the Riemann surface $R$.

Proposition 1.1. Every element $f \in \operatorname{Cov}(R)-\operatorname{Conf}(R)$ induces an element $f^{*}$ belonging to $\operatorname{Emb}_{0}(T(R))-\operatorname{Aut}_{0}(T(R))$.

Indeed, this assertion is based on a fact that a holomorphic cover of Riemann surfaces induces an injective holomorphic map between the corresponding Teichmüller spaces, where the condition of preserving the infinity can be easily verified if we consider the Bers embeddings of their Teichmüller spaces. We call such an element $f^{*} \in \operatorname{Emb}_{0}(T(R))$ induced by $f \in \operatorname{Cov}(R)$ geometric, and denote by $\operatorname{Cov}^{*}(T(R))$ the sub-semigroup of $\operatorname{Emb}_{0}(T(R))$ consisting of all geometric elements.

We should emphasize again that the fact that $\operatorname{Emb}_{0}(T(R))-\operatorname{Aut}_{0}(T(R))$ is not empty is far from trivial, which is one of the amazing phenomena for 
infinite dimensional Teichmüller spaces. This follows from non-emptiness of the corresponding set $\operatorname{Cov}(R)-\operatorname{Conf}(R)$. However, there seems no systematic researches on this subject matter before. Thus our first work is to investigate the structure of non-injective holomorphic self-covers of Riemann surfaces, and to show that they are not empty for many Riemann surfaces.

Theorem 1.2. If a Riemann surface $R$ with a non-cyclic fundamental group admits a holomorphic self-cover $f \in \operatorname{Cov}(R)-\operatorname{Conf}(R)$, then there exist a holomorphic cover $f_{\infty}: R \rightarrow R_{\infty}$ and a conformal automorphism $g_{\infty} \in \operatorname{Conf}\left(R_{\infty}\right)$ of infinite order such that $f_{\infty} \circ f=g_{\infty} \circ f_{\infty}$. Conversely, for a Riemann surface $\underline{R}$ with a conformal automorphism $g \in \operatorname{Conf}(\underline{R})$ of infinite order, there exist a holomorphic cover $f: R \rightarrow \underline{\bar{R}}$ and a holomorphic self-cover $f \in \operatorname{Cov}(R)-\operatorname{Conf}(R)$ such that $\underline{f} \circ f=\underline{g} \circ \underline{f}$.

Here we give an example of a holomorphic non-injective self-cover.

Example 1.3. A pair of pants is a hyperbolic surface homeomorphic to a three-punctured sphere having three geodesic boundary components. Choose a pair of pants $P$ whose boundary components $c_{0}, c_{1}$ and $c_{2}$ have the same length. First, glue two copies of $P$ along the 2 boundary components $c_{1}$ and $c_{2}$ of $P$, which results in a hyperbolic surface $P_{1}$ with 5 boundary components. Next, glue four copies of $P$ along the 4 boundary components of $P_{1}$ coming from $c_{1}$ and $c_{2}$, which results in a hyperbolic surface $P_{2}$ with 9 boundary components. Continuing this process infinitely many times, we have a hyperbolic surface $P_{\infty}$ with the boundary component $c_{0}$. Let $\Gamma$ be a Fuchsian group acting on the unit disk $\Delta$ such that $R=\Delta / \Gamma$ is the Nielsen extension of $P_{\infty}$ beyond $c_{0}$. On the other hand, for a connected component $R^{\prime}$ of $P_{\infty}-P$, the subgroup $\Gamma^{\prime}$ of $\Gamma \cong \pi_{1}(R)$ corresponding to $\pi_{1}\left(R^{\prime}\right)$ is properly contained in $\Gamma$ but it is conformally conjugate to $\Gamma$. This implies that the Riemann surface $R$ admits a holomorphic non-injective self-cover.

The Teichmüller space $T(R)$ is endowed with the Teichmüller-Kobayashi distance $d_{T(R)}$ and every holomorphic self-map $F$ of $T(R)$ is not expanding with respect to this distance. A geometric self-embedding $F=f^{*} \in \operatorname{Cov}^{*}(T(R))$ is not necessarily isometric, but it is close to an isometry in the following sense, namely, $f^{*}$ is a strongly bounded contraction with respect to $d_{T(R)}$.

Proposition 1.4. For any geometric self-embedding $f^{*} \in \operatorname{Cov}^{*}(T(R))$, there exists a uniform constant $c>0$ such that

$$
c d_{T(R)}(p, q) \leq d_{T(R)}\left(\left(f^{*}\right)^{n}(p),\left(f^{*}\right)^{n}(q)\right) \leq d_{T(R)}(p, q)
$$

for any $p$ and $q$ in $T(R)$ and for any $n \in \mathbf{N}$.

Next, we consider the dynamics on $T(R)$ induced by the iteration of $f^{*} \in$ $\operatorname{Cov}^{*}(T(R))$ and introduce the concept of the full cluster set, the recurrent set 
and the limit set. As the following proposition implies, this case contrasts with the case where $F \in \operatorname{End}_{0}(T(R))$ is strictly contracting and $T(R)$ shrinks to the unique attracting fixed point of $F$ by the iteration of $F$.

Proposition 1.5. For every geometric self-embedding $f^{*} \in \operatorname{Cov}^{*}(T(R))$, the full cluster set $C\left(f^{*}\right)=\bigcap_{n=1}^{\infty}\left(f^{*}\right)^{n}(T(R))$ is a non-degenerate submanifold of $T(R)$, which can be identified with the Teichmüller space $T\left(R_{\infty}\right)$ for a Riemann surface $R_{\infty}$ by a holomorphic cover $R \rightarrow R_{\infty}$. The recurrent set $\operatorname{Rec}\left(f^{*}\right)$ and the limit set $\Lambda\left(f^{*}\right)$ are coincident and contained in $C\left(f^{*}\right)$.

However, even on this $C\left(f^{*}\right)$, the distribution of contracting/isometric tangent vectors by the derivative $d f^{*}$ of $f^{*} \in \mathrm{Cov}^{*}(T(R))$ is not so clear. Actually, the isometry locus of $d f^{*}$ on the holomorphic tangent bundle over $T(R)$ can be a nowhere dense closed subset and, at the same time, non-empty.

Theorem 1.6. For a non-amenable holomorphic self-cover $f$, the set of tangent vectors strictly contracted by $d f^{*}$ is open and dense in the holomorphic tangent bundle $T(T(R))$. On the other hand, the projection of the isometric locus of $d f^{*}$ in $T(T(R))$ to $T(R)$ contains the recurrent set $\operatorname{Rec}\left(f^{*}\right)$.

Here, the assumption that $f$ is a non-amenable cover is essential according to McMullen [14]. In fact, for every amenable holomorphic self-cover $f$ of $R$, the derivative $d f^{*}$ is isometric on the whole $T(T(R))$.

In the previous paragraphs, we restrict ourselves to the self-embeddings of $T(R)$ fixing the base point. When we remove this restriction, the corresponding self-maps of $R$ will be quasiregular self-covers of $R$. Here a quasiregular cover means the composition of a holomorphic cover and a quasiconformal homeomorphism. We denote the semigroup consisting of all quasiregular self-covers of $R$ by $\mathrm{QCov}(R)$. Accordingly, the sub-semigroup of $\operatorname{Emb}(T(R))$ consisting of all geometric elements induced by quasiregular self-covers of $R$ is denoted by $\mathrm{QCov}^{*}(T(R))$. The central problem for this subject is to determine whether $\operatorname{Emb}(T(R))$ is coincident with $\operatorname{QCov}^{*}(T(R))$ or not. This would be a generalization of the fact that $\operatorname{Aut}(T(R))$ is coincident with $\mathrm{QC}^{*}(T(R))$, which is identified with $\operatorname{MCG}(R)$. Although we cannot prove or disprove it so far, we will seek a way of investigating this problem by using invariant metrics on the Teichmüller space.

Dynamics on $T(R)$ by $f^{*} \in \mathrm{QCov}^{*}(T(R))$ are essentially the same as those by elements of $\operatorname{Cov}^{*}(T(R))$ if $f^{*}$ has a fixed point on $T(R)$. This is the case if the quasiregular self-cover $f$ is quasiconformally conjugate to a holomorphic self-cover. One may consider the problem of finding the fixed point as a generalization of the Nielsen realization problem on a certain mapping class group. We consider equivalent conditions to the existence of fixed points and obtain the following assertion. Similar situations often appear in the study of 
complex dynamics of rational maps and our result is related to those subject matters.

Theorem 1.7. A quasiregular self-cover $f \in \mathrm{Q} \operatorname{Cov}(R)$ is equivalent to a holomorphic self-cover of another Riemann surface $R^{\prime}$ by quasiconformal homeomorphisms $R \rightarrow R^{\prime}$ in the same Teichmüller class if and only if the orbit of $f^{*} \in \mathrm{QCov}^{*}(T(R))$ is bounded in $T(R)$.

A brief summary of this paper is as follows. In Section 2, we give the fundamental structure theorem for holomorphic self-covering of $R$ and show that $\operatorname{Cov}(R)-\operatorname{Conf}(R)$ is non-empty for fairly general $R$. In Section 3, for a holomorphic self-embedding $F \in \operatorname{Emb}(T(R))$ satisfying a certain condition, an $F$-invariant distance on $T(R)$ is constructed and the strongly bounded property is proved. Every geometric self-embedding $f^{*} \in \operatorname{QCov}^{*}(T(R))$ satisfies that condition. In Section 4 , we clarify the full cluster set $C\left(f^{*}\right)$ for the dynamics $f^{*} \in \operatorname{Cov}^{*}(T(R))$ on $T(R)$ as an application of the structure theorem for holomorphic self-covering. In Section 5, we extend the structure theorem to the quasiregular self-covers $\mathrm{Q} \operatorname{Cov}(R)$ and give an answer to the fixed point problem for such maps. Finally in Section 6, we prove that any element $f^{*} \in \operatorname{Cov}^{*}(T(R))$ is strictly contracting almost everywhere in the holomorphic tangent bundle over $T(R)$ if $f$ is a non-amenable holomorphic self-cover of $R$.

\section{StRuCTURE THEOREM FOR NON-INJECTIVE SELF-COVERINGS OF RIEMANN SURFACES}

We always assume that a Riemann surface $R$ admits a hyperbolic metric and has a non-cyclic fundamental group. Namely, $R$ is represented as the quotient space $\Delta / \Gamma$ of the unit disk $\Delta \subset \mathbf{C}$ by a non-elementary torsion-free Fuchsian group $\Gamma$. We denote by $\operatorname{Cov}(R)$ the semigroup of all holomorphic self-covers of a Riemann surfaces $R$. We assume that $R$ has a non-injective holomorphic self-cover $f: R \rightarrow R$. Hereafter in this paper, covering is always meant to be unlimited and unbranched unless we specifically mention otherwise.

First, we prove the following structure theorem of self-covering, which is a basis of our consideration on the dynamics of holomorphic self-covers on Riemann surfaces. Similar results have appeared in Jørgensen, Marden and Pommerenke [9], Beardon [1] and McMullen and Sullivan [16].

Theorem 2.1. Let $R$ be a Riemann surface, $\pi: \Delta \rightarrow R$ a holomorphic universal cover and $\Gamma \subset \operatorname{Aut}(\Delta)$ the covering transformation group for $\pi$, which is a non-elementary torsion-free Fuchsian group. Suppose that $R$ has a noninjective holomorphic self-cover $f: R \rightarrow R$. Then the following claims are satisfied. 
(1) There exists a conformal automorphism $g \in \operatorname{Aut}(\Delta)$ such that $f \circ \pi=$ $\pi \circ g$. The conjugate $\Gamma_{1}=g^{-1} \Gamma g$ properly contains $\Gamma$, which is the covering transformation group for $f \circ \pi$.

(2) Set $\Gamma_{n}=g^{-n} \Gamma g^{n}$ for each $n \in \mathbf{N}$. They are the covering transformation groups for $f^{n} \circ \pi$ and the following proper inclusion relations hold.

$$
\Gamma=\Gamma_{0} \varsubsetneqq \Gamma_{1} \varsubsetneqq \cdots \varsubsetneqq \Gamma_{n} \varsubsetneqq \cdots
$$

(3) Let $\Gamma_{\infty}=\bigcup_{n=0}^{\infty} \Gamma_{n}=\lim _{n \rightarrow \infty} \Gamma_{n}$. Then $\Gamma_{\infty}$ is discrete and torsion-free. Actually it is the geometric limit of the sequence $\left\{\Gamma_{n}\right\}$.

(4) The conformal automorphism $g \in \operatorname{Aut}(\Delta)$ belongs to the normalizer of the Fuchsian group $\Gamma_{\infty}$, that is, $g^{-1} \Gamma_{\infty} g=\Gamma_{\infty}$. Let $R_{\infty}=\Delta / \Gamma_{\infty}$ be the Riemann surface represented by $\Gamma_{\infty}$ and let $g_{\infty}$ be the conformal automorphism of $R_{\infty}$ induced by $g$. Then $g_{\infty}$ is of infinite order and $R_{\infty}$ is of topologically infinite type.

(5) Let $f_{\infty}: R \rightarrow R_{\infty}$ be the holomorphic cover corresponding to the inclusion relation $\Gamma \subset \Gamma_{\infty}$. Then it satisfies $g_{\infty} \circ f_{\infty}=f_{\infty} \circ f$. In fact, the following diagram commutes:

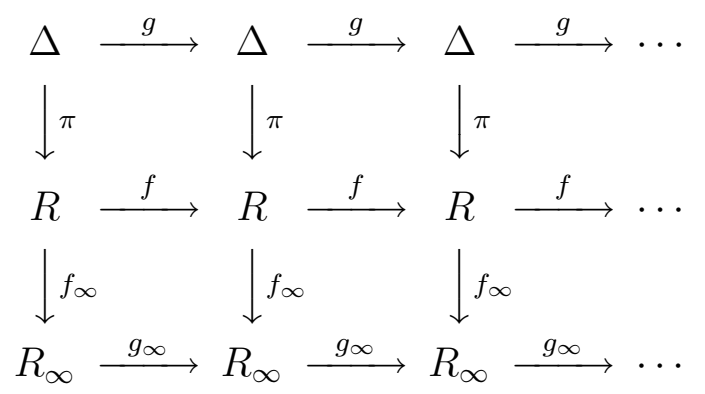

(6) Let $\hat{\Gamma}=\langle\Gamma, g\rangle$ be a subgroup of $\operatorname{Aut}(\Delta)$ generated by $\Gamma$ and $g$. Then $\hat{\Gamma}$ is a torsion-free Fuchsian group and is represented as a semi-direct product $\hat{\Gamma}=\Gamma_{\infty} \rtimes\langle g\rangle$. The quotient $R_{\infty} /\left\langle g_{\infty}\right\rangle$ of $R_{\infty}$ by the cyclic group of the conformal automorphism $g_{\infty}$ is coincident with the Riemann surface $\hat{R}=\Delta / \hat{\Gamma}$.

(7) Suppose that there are a holomorphic cover $f: R \rightarrow \underline{R}$ and a biholomorphic automorphism $\underline{g}: \underline{R} \rightarrow \underline{R}$ of a Riemann surface $\underline{R}$ satisfying $g \circ f=f \circ f$. Then there is a holomorphic cover $\hat{f}: R_{\infty} \rightarrow \underline{R}$ such that $g \circ \overline{\hat{f}}=\overline{\hat{f}} \circ g_{\infty}$. In other words, $f_{\infty}: R \rightarrow R_{\infty}$ is the highest holomorphic cover from $R$ among all such $\underline{f}: R \rightarrow \underline{R}$.

Proof. Most of the statements follows in line-by-line order from general facts on covering of Riemann surfaces and its transformation groups. Here we give proofs only for less trivial statements (3) and (4). 
Concerning statement (3), the discreteness of $\Gamma_{\infty}$ follows from the Jørgensen criterion [8], which asserts that a non-elementary group $G$ of Möbius transformations is discrete if and only if the subgroup $\left\langle g_{1}, g_{2}\right\rangle$ is discrete for all pairs of elements $g_{1}$ and $g_{2}$ in $G$. Furthermore, since all $\Gamma_{n}$ have no torsion elements, so does $\Gamma_{\infty}$. It is easy to see that the increasing sequence $\left\{\Gamma_{n}\right\}$ has the geometric limit $\Gamma_{\infty}^{\prime}$ which contains $\Gamma_{\infty}$. However, since $\Gamma_{\infty}$ is discrete, they are coincident.

For statement (4), first we show that $g^{-1} \Gamma_{\infty} g=\Gamma_{\infty}$. Indeed, the inclusion $g^{-1} \Gamma_{\infty} g \subset \Gamma_{\infty}$ follows from $g^{-1} \Gamma_{n} g=\Gamma_{n+1} \subset \Gamma_{\infty}$ and the other inclusion $g \Gamma_{\infty} g^{-1} \subset \Gamma_{\infty}$ follows from $g \Gamma_{n} g^{-1}=\Gamma_{n-1} \subset \Gamma_{\infty}$. Next we show that $g_{\infty}$ is of infinite order. Indeed, $g_{\infty}^{m}=i d$ for some $m \in \mathbf{Z}$ implies that $g^{m} \in \Gamma_{\infty}$, and hence $g^{m} \in \Gamma_{n}$ for some $n$. Then $f^{m}=i d$, but this is impossible since $f$ is not injective.

In the diagram of Theorem 2.1, we assume the top-left $\Delta$ as the base stage. Then we have a diagram of the covering transformation groups action on this $\Delta$ for the corresponding covering maps:

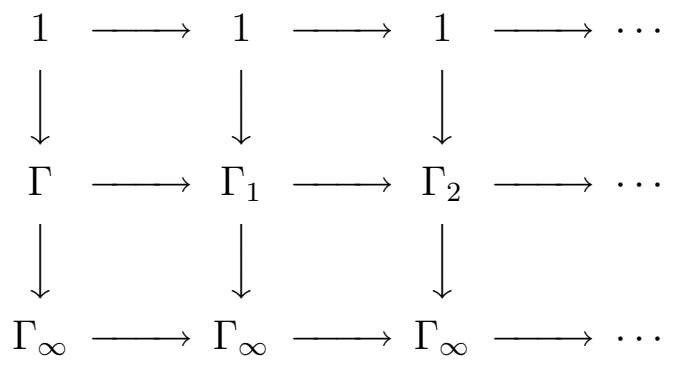

Here all the arrows indicate the inclusion maps.

Remark 2.2. Theorem 2.1 is not valid when the fundamental group of $R$ is cyclic. We will consider this case when $R$ is a punctured disk. The corresponding Fuchsian group $\Gamma$ is a parabolic cyclic group. We may assume that $\Gamma$ acts on the upper half-plane $H$ and is generated by $\gamma(z)=z+1$. Take $g(z)=k z$ for some positive integer $k$ and take the conjugate $\Gamma_{1}=g^{-1} \Gamma g$, which is a cyclic group generated by $\gamma_{1}(z)=z+1 / k$. This defines a holomorphic $k$-sheeted self-cover $f: R=H / \Gamma \rightarrow R=H / \Gamma_{1}$. Moreover, $\Gamma_{n}=g^{-n} \Gamma g^{n}(n \in \mathbf{N})$ gives an increasing sequence with the geometric limit $\Gamma_{\infty}=\lim \Gamma_{n}$. It is easy to see that $\Gamma_{\infty}$ consists of all parabolic transformations $z \mapsto z+\alpha$ where $\alpha$ is any $k$-adic rational number. Hence $\Gamma_{\infty}$ is not discrete but is elementary. It is the same for $\hat{\Gamma}=\langle\gamma, g\rangle$.

Theorem 2.1 tells us that, for the investigation of the action of the self-cover $f$ on $R$, it is helpful to see the action of the automorphism $g_{\infty}$ of $R_{\infty}$. On the other hand, the orbit of $f$ itself defines the following equivalence relations, which are also important. See also [16, Section 6]. 
Definition 2.3. Let $f: R \rightarrow R$ be a map in general. The grand orbit of $x \in R$ under $f$ is the set of all points $x^{\prime} \in R$ such that $f^{n}(x)=f^{m}\left(x^{\prime}\right)$ for some $n \geq 0$ and $m \geq 0$, which is denoted by $\hat{O}(f, x)$. The small orbit of $x \in R$ under $f$ is the set of all points $x^{\prime} \in R$ such that $f^{n}(x)=f^{n}\left(x^{\prime}\right)$ for some $n \geq 0$, which is denoted by $O(f, x)$. Clearly $O(f, x) \subset \hat{O}(f, x)$. The equivalence relation defined by the grand orbit under $f$ is called the grand orbit equivalence relation for $f$ and is denoted by $x \approx_{f} x^{\prime}$ when $x$ and $x^{\prime}$ are grand orbit equivalent. The equivalence relation defined by the small orbit under $f$ is called the small orbit equivalence relation for $f$ and is denoted by $x \sim_{f} x^{\prime}$ when $x$ and $x^{\prime}$ are small orbit equivalent.

Here we note the relationship between these two equivalence relations and the action of the automorphism $g_{\infty}$ of $R_{\infty}$.

Proposition 2.4. The quotient space $R / \sim_{f}$ by the small orbit equivalence relation for $f$ is coincident with the Riemann surface $R_{\infty}=\Delta / \Gamma_{\infty}$. The quotient space $R / \approx_{f}$ by the grand orbit equivalence relation for $f$ is coincident with the Riemann surface $\hat{R}=\Delta / \hat{\Gamma}$.

Proof. Take two points $z$ and $z^{\prime}$ in $\Delta$. If $x=\pi(z)$ and $x^{\prime}=\pi\left(z^{\prime}\right)$ in $R$ are in the same small orbit, then there is some $n \geq 0$ such that $f^{n}(\pi(z))=f^{n}\left(\pi\left(z^{\prime}\right)\right)$.

Since $\Gamma_{n}$ is the covering transformation group for $f^{n} \circ \pi$, this implies that $z$ and $z^{\prime}$ are equivalent under $\Gamma_{n}$, and hence under $\Gamma_{\infty}$. Conversely, if $z$ and $z^{\prime}$ are equivalent under $\Gamma_{\infty}$, then there is some $n \geq 0$ such that they are equivalent under $\Gamma_{n}$. Hence $f^{n}(\pi(z))=f^{n}\left(\pi\left(z^{\prime}\right)\right)$ and $x$ and $x^{\prime}$ are in the same small orbit.

Also, if $x=\pi(z)$ and $x^{\prime}=\pi\left(z^{\prime}\right)$ in $R$ are in the same grand orbit, then there is some $n \geq 0$ and $m \geq 0$ such that $f^{n}(\pi(z))=f^{m}\left(\pi\left(z^{\prime}\right)\right)$. We may assume that $m \geq n$. Since $f^{m-n} \circ \pi=\pi \circ g^{m-n}$, this yields $f^{n}(\pi(z))=f^{n}\left(\pi\left(g^{m-n}\left(z^{\prime}\right)\right)\right)$. Hence $z$ and $g^{m-n}\left(z^{\prime}\right)$ are equivalent under $\Gamma_{n}$, and thus $z$ and $z^{\prime}$ are equivalent under $\hat{\Gamma}$. The converse direction can be seen by the fact that any element $\hat{\gamma} \in \hat{\Gamma}=\Gamma_{\infty} \rtimes\langle g\rangle$ has a representation $\hat{\gamma}=\gamma g^{k}\left(\gamma \in \Gamma_{\infty}\right)$.

Example 2.5. Let $f$ be a rational map of the Riemann sphere that has an immediate attracting or parabolic basin $D$. Suppose that the grand orbit $\hat{O}$ of the critical points of $f$ is discrete in $D$ or $f$ has a non-critical attracting fixed point in $D$. We consider a Riemann surface $R=D-\operatorname{cl}(\hat{O})$. The restriction of $f$ to $R$ gives a finite-sheeted holomorphic self-cover and $\hat{R}=R / \approx_{f}$ is an analytically finite Riemann surface (see [16]). For instance, consider $f(z)=$ $z^{2}+1 / 4$, which has an immediate parabolic basin $D$. In this case, $f: R \rightarrow R$ is a two-sheeted normal cover and the quotient $\hat{R}$ is a three-punctured sphere.

In the last of this section, we prove that an element of $\operatorname{Cov}(R)-\operatorname{Conf}(R)$ exists widely. 
Theorem 2.6. For every Riemann surface $\underline{R}$ with a conformal automorphism $\underline{g}: \underline{R} \rightarrow \underline{R}$ of infinite order, there exist a holomorphic cover $\underline{f}: R \rightarrow \underline{R}$ and $a$ non-injective holomorphic self-cover $f: R \rightarrow R$ such that $\underline{f} \circ \bar{f}=\underline{g} \circ \underline{f}$.

For the proof of Theorem 2.6, we note the following proposition, which concerns the fundamental groups of surfaces. See [9].

Proposition 2.7. Let $\hat{\Gamma}$ be a Fuchsian group represented by $\hat{\Gamma}=\Gamma_{\infty} \rtimes\langle g\rangle$. Then there exist a proper subgroup $H \varsubsetneqq \Gamma_{\infty}$ and subgroups $J_{i} \subset H(i=0,1)$ such that $\hat{\Gamma}$ is the $H N N$-extension $H *_{g_{0}}$ of $H$ by some element $g_{0}$ in the coset $g \Gamma_{\infty}$ satisfying $g_{0} J_{0} g_{0}^{-1}=J_{1}$.

Proof. Let $\hat{R}=\Delta / \hat{\Gamma}$ and $R_{\infty}=\Delta / \Gamma_{\infty}$. Let $g_{\infty}$ be the conformal automorphism of $R_{\infty}$ induced by $g$. Taking a base point $x_{0} \in R_{\infty}$ arbitrarily, we have a Dirichlet fundamental domain $W$ of $\left\langle g_{\infty}^{2}\right\rangle$ in $R_{\infty}$ centered at $x_{0}$. Namely,

$$
W=\left\{x \in R_{\infty} \mid d_{R_{\infty}}\left(x, x_{0}\right)<d_{R_{\infty}}\left(x, g_{\infty}^{2 k}\left(x_{0}\right)\right) \text { for all } k \in \mathbf{Z}-\{0\}\right\},
$$

where $d_{R_{\infty}}$ denotes the hyperbolic distance on $R_{\infty}$. Let $V_{0}=W \cap g_{\infty}^{-1}(W)$ and $V_{1}=g_{\infty}\left(V_{0}\right)$. Since the identification on $W$ by $g_{\infty}$ gives the Riemann surface $\hat{R}=R_{\infty} /\left\langle g_{\infty}\right\rangle$, the subgroups $H, J_{0}$ and $J_{1}$ of $\Gamma_{\infty} \cong \pi_{1}\left(R_{\infty}\right)$ corresponding to the fundamental groups of $W, V_{0}$ and $V_{1}$ respectively give the representation of $\hat{\Gamma} \cong \pi_{1}(\hat{R})$ by the HNN-extension.

Proof of Theorem 2.6. We represent $\underline{R}=\Delta / \underline{\Gamma}$ by a Fuchsian group $\underline{\Gamma}$ and take a lift $g \in \operatorname{Aut}(\Delta)$ of $g$ such that $g^{-1} \underline{\Gamma} g=\underline{\Gamma}$. Then by Proposition 2.7, there exists a proper subgroup $H \varsubsetneqq \underline{\Gamma}$ such that $\hat{\Gamma}:=\underline{\Gamma} \rtimes\langle g\rangle$ is the HNN-extension of $H$. Set

$$
\Gamma=\left\langle g^{\ell} h g^{-\ell}: h \in H, \ell \geq 0\right\rangle,
$$

which is a proper subgroup of $\underline{\Gamma}$. Then, for the Riemann surface $R=\Delta / \Gamma$, we have a holomorphic cover $\underline{f}: R \rightarrow \underline{R}$.

Moreover, set

$$
\Gamma_{n}=\left\langle g^{\ell} h g^{-\ell}: h \in H, \ell \geq-n\right\rangle,
$$

which is coincident with $g^{-n} \Gamma g^{n}$. It is clear that

$$
\Gamma \subset \Gamma_{1} \subset \Gamma_{2} \subset \cdots \subset \underline{\Gamma} \text {. }
$$

This implies that there is a holomorphic self-cover $f: R \rightarrow R$ that satisfies $\underline{f} \circ f=\underline{g} \circ \underline{f}$.

The limit $\Gamma_{\infty}=\bigcup \Gamma_{n}$ is a subgroup of $\underline{\Gamma}$ represented by

$$
\Gamma_{\infty}=\left\langle g^{\ell} h g^{-\ell}: h \in H, \ell \in \mathbf{Z}\right\rangle .
$$

Since $g^{-1} \Gamma_{\infty} g=\Gamma_{\infty}$ and $\left\langle\Gamma_{\infty}, g\right\rangle=\hat{\Gamma}$, we see that $\hat{\Gamma}=\Gamma_{\infty} \rtimes\langle g\rangle$, from which $\Gamma_{\infty}=\underline{\Gamma}$ follows. Hence the inclusion relations of $\left\{\Gamma_{n}\right\}$ are proper, which implies that the covering map $f$ is non-injective. 


\section{Holomorphic SelF-embeddings of Teichmüller SPACES AND BOUNDED CONTRACTION PROPERTY}

In this section, we consider holomorphic self-embeddings of the Teichmüller space and their bounded contraction property with respect to the Teichmüller distance.

The Teichmüller space $T(R)$ of a Riemann surface $R=\Delta / \Gamma$ is the set of equivalence classes $[f]$ of quasiconformal homeomorphisms $f$ of $R$. Here we say that two quasiconformal homeomorphisms $f_{1}$ and $f_{2}$ of $R$ are Teichmüller equivalent if there exists a conformal homeomorphism $h: f_{1}(R) \rightarrow f_{2}(R)$ such that $f_{2}^{-1} \circ h \circ f_{1}$ is homotopic to the identity of $R$. Here the homotopy is considered to be relative to the ideal boundary at infinity of $R$. One can consult monographs [5], [6], [7], [11] and [18] for basic facts on Teichmüller spaces mentioned hereafter.

A distance between two points $\left[f_{1}\right]$ and $\left[f_{2}\right]$ in $T(R)$ is defined by

$$
d_{T(R)}\left(\left[f_{1}\right],\left[f_{2}\right]\right)=\frac{1}{2} \log K(f),
$$

where $f$ is an extremal quasiconformal homeomorphism in the sense that its maximal dilatation $K(f)$ is minimal in the homotopy class of $f_{2} \circ f_{1}^{-1}$. Then $d_{T(R)}$ is a complete distance on $T(R)$ which is called the Teichmüller distance.

Let $\Delta^{*}$ be the complement of $\bar{\Delta}$ in the Riemann sphere and $B(\Gamma)$ the complex Banach space of all bounded holomorphic quadratic differentials for $\Gamma$ on $\Delta^{*}$ endowed with the hyperbolic supremum norm. Then the Teichmüller space $T(R)$ is a complex Banach manifold modeled on $B(\Gamma)$. In fact, $T(R)$ is embedded in $B(\Gamma)$ as a bounded contractible domain $T_{B}(\Gamma)$. More precisely, for a holomorphic universal cover $\pi: \Delta \rightarrow R$, we have an injection $\beta_{\pi}: T(R) \rightarrow B(\Gamma)$ whose image is $T_{B}(\Gamma)$. This is called the Bers embedding of $T(R)$. If $R$ is analytically infinite, then $T(R)$ is infinite dimensional, and vice versa.

The Teichmüller distance $d_{T(R)}$ is coincident with the Kobayashi distance on the complex manifold $T(R)$ for every Riemann surface (see [5]). The Kobayashi distance has the non-expanding property for holomorphic maps. Concerning the Kobayashi distance, one can refer to [10].

We denote by $\operatorname{End}(T(R))$ the semigroup of all holomorphic self-maps of $T(R)$ and by $\operatorname{End}_{0}(T(R))$ the sub-semigroup of $\operatorname{End}(T(R))$ consisting the elements fixing the base point $o=[i d]$ of $T(R)$. Moreover, we denote by $\operatorname{Emb}(T(R))$ the semigroup consisting of all injective holomorphic self-maps of $T(R)$ preserving the infinity and define its sub-semigroup $\operatorname{Emb}_{0}(T(R))=$ $\operatorname{Emb}(T(R)) \cap \operatorname{End}_{0}(T(R))$. Here we say that $F \in \operatorname{End}(T(R))$ preserves the infinity if the following condition is satisfied: 
$\left(^{*}\right) F(p)$ tends to the infinity of $T(R)$, that is, $F(p)$ escapes from each bounded set of $T(R)$ if and only if $p$ tends to the infinity.

Of course, this definition can be extended to any map between metric spaces. We call an element in $\operatorname{Emb}(T(R))$ a holomorphic self-embedding of $T(R)$.

Every holomorphic cover $f: R \rightarrow R^{\prime}$ of a Riemann surface $R$ onto another Riemann surface $R^{\prime}$ induces an injective holomorphic map $f^{*}: T\left(R^{\prime}\right) \rightarrow T(R)$ between their Teichmüller spaces that preserves the base points. If we consider the Bers embeddings $T_{B}\left(\Gamma^{\prime}\right)$ and $T_{B}(\Gamma)$ for $T\left(R^{\prime}\right)$ and $T(R)$ respectively, then $f^{*}$ is nothing but the inclusion map $T_{B}\left(\Gamma^{\prime}\right) \hookrightarrow T_{B}(\Gamma)$.

We consider a Riemann surface $R$ that admits a holomorphic self-cover $f \in$ $\operatorname{Cov}(R)$. It induces an injective holomorphic self-map $f^{*}$ of $T(R)$ preserving the base point. We call such an element geometric and denote by $\operatorname{Cov}^{*}(T(R))$ the sub-semigroup of $\operatorname{End}_{0}(T(R))$ consisting of all geometric elements. By considering the Bers embeddings, we see that $f^{*} \in \operatorname{Cov}^{*}(T(R))$ preserves the infinity, namely, $\operatorname{Cov}^{*}(T(R)) \subset \operatorname{Emb}_{0}(T(R))$.

A quasiregular map is the composition of a quasiconformal homeomorphism and a holomorphic map. A quasiregular cover is a covering map given by a quasiregular map. Every quasiregular cover $f: R \rightarrow R^{\prime}$ of a Riemann surface $R$ onto another Riemann surface $R^{\prime}$ induces an injective holomorphic map $f^{*}$ : $T\left(R^{\prime}\right) \rightarrow T(R)$ between their Teichmüller spaces, not necessarily preserving the base points. This is a generalization of the fact that a quasiconformal homeomorphism $h: R \rightarrow R^{\prime}$ induces a biholomorphic homeomorphism $h^{*}$ : $T\left(R^{\prime}\right) \rightarrow T(R)$. By [12], we see that $f$ is injective if and only if $f^{*}$ is surjective.

Similar to the case of holomorphic self-covering, we consider a Riemann surface $R$ that admits a quasiregular cover $f$ of $R$ onto itself. We call such an $f$ quasiregular self-cover and denote the set of all quasiregular self-covers of $R$ by QCov $(R)$. Every quasiregular self-cover of $R$ induces an injective holomorphic self-map $f^{*} \in \operatorname{End}(T(R))$. We also call such an element geometric and denote by $\mathrm{QCov}^{*}(T(R))$ the sub-semigroup of $\operatorname{End}(T(R))$ consisting of all geometric elements. As is seen later, $f^{*} \in \mathrm{QCov}^{*}(T(R))$ preserves the infinity, namely, $\mathrm{QCov}^{*}(T(R)) \subset \operatorname{Emb}(T(R))$.

In this section, we investigate the inclusion $\operatorname{QCov}^{*}(T(R)) \subset \operatorname{Emb}(T(R))$ more closely. We denote by $d_{T(\Delta)}$ the universal Teichmüller distance on $T(\Delta)$ as well as the induced distance on $T(R)$ by the embedding $\pi^{*}: T(R) \rightarrow T(\Delta)$. The non-expanding property of $\pi^{*}$ implies $d_{T(\Delta)} \leq d_{T(R)}$. For a holomorphic self-map $F \in \operatorname{End}(T(R))$, we consider the following condition:

(**) There exists a distance $d_{0}$ on $T(R)$ with $d_{0} \leq d_{T(R)}$ such that $F$ is an isometry with respect to $d_{0}$.

In what follows, we call a distance on $T(R)$ for which a map $F: T(R) \rightarrow$ $T(R)$ is isometric an $F$-invariant distance. Also, if $F$ satisfies condition $(* *)$, then we say that $F$ is virtually isometric. We will see in Section 5 that every 
geometric element $f^{*} \in \mathrm{QCov}^{*}(T(R))$ is isometric with respect to $d_{T(\Delta)}$ and thus virtually isometric.

In this section, we prove that a holomorphic self-map $F$ of $T(R)$ that is virtually isometric is a strongly bounded contraction in the following sense.

Definition 3.1. A holomorphic self-map $F \in \operatorname{End}(T(R))$ is said to be a bounded contraction with respect to $d_{T(R)}$ if there exists a uniform constant $c>0$ such that

$$
c d_{T(R)}(p, q) \leq d_{T(R)}(F(p), F(q)) \leq d_{T(R)}(p, q)
$$

for any $p$ and $q$ in $T(R)$. Moreover, $F \in \operatorname{End}(T(R))$ is said to be a strongly bounded contraction if there exists a uniform constant $c>0$ such that

$$
c d_{T(R)}(p, q) \leq d_{T(R)}\left(F^{n}(p), F^{n}(q)\right) \leq d_{T(R)}(p, q)
$$

for any $p$ and $q$ in $T(R)$ and for any $n \in \mathbf{N}$.

Theorem 3.2. Every virtually isometric, holomorphic self-map $F \in \operatorname{End}(T(R))$ is a strongly bounded contraction with respect to $d_{T(R)}$.

Clearly, if $F \in \operatorname{End}(T(R))$ is a bounded contraction, then it preserves the infinity, namely $F \in \operatorname{Emb}(T(R))$. As is mentioned in the introduction, our future problem is to show that $\operatorname{QCov}^{*}(T(R))=\operatorname{Emb}(T(R))$. As a weaker version of this problem, we are also interested in a question whether a virtually isometric, holomorphic self-map is geometric or not.

To prove Theorem 3.2, we introduce another $F$-invariant distance as follows.

Definition 3.3. For any non-expanding map $F: T(R) \rightarrow T(R)$ with respect to $d_{T(R)}$, a pseudo-distance $d_{F}$ is defined by

$$
d_{F}(p, q)=\lim _{n \rightarrow \infty} d_{T(R)}\left(F^{n}(p), F^{n}(q)\right)
$$

for any $p$ and $q$ in $T(R)$.

Let $d$ be a distance on $T(R)$ that is topologically equivalent to $d_{T(R)}$. For any smooth curve $\alpha:[0,1] \rightarrow T(R)$, the length of $\alpha$ with respect to $d$ is denoted by $L_{d}(\alpha)$. The inner distance $d^{i}$ induced by $d$ is the path distance defined by the infimum of the lengths $L_{d}(\alpha)$ taken over all smooth curves $\alpha$ connecting two points. Then $d^{i} \geq d$ holds in general. When the equality is satisfied, the distance $d$ is called an inner distance. Since the Teichmüller distance $d_{T(R)}$ is the Kobayashi distance on $T(R)$, it is an inner distance. See [10].

Proposition 3.4. For a virtually isometric, holomorphic self-map $F: T(R) \rightarrow$ $T(R)$, the pseudo-distance $d_{F}$ is a distance. It is the maximal distance among all $F$-invariant distances $d$ satisfying $d \leq d_{T(R)}$. Moreover, it is an inner distance on $T(R)$. 
Proof. The invariance of $d_{F}$ under $F$ is clear from the definition. Every $F$ invariant distance $d$ with $d \leq d_{T(R)}$ satisfies

$$
d_{T(R)}\left(F^{n}(p), F^{n}(q)\right) \geq d\left(F^{n}(p), F^{n}(q)\right)=d(p, q)
$$

for any $p$ and $q$ in $T(R)$ and for any $n \in \mathbf{N}$. This yields $d_{F} \geq d$ and, since there exists at least one $F$-invariant distance $d_{0}\left(\leq d_{T(R)}\right)$ by being virtually isometric, $d_{F}$ is the maximal distance.

The inner distance $d_{F}^{i}$ induced by $d_{F}$ is also $F$-invariant. Since $d_{F} \leq d_{T(R)}$ and $d_{T(R)}^{i}=d_{T(R)}$, we see that $d_{F}^{i} \leq d_{T(R)}$. Then the maximality of $d_{F}$ yields $d_{F}^{i}=d_{F}$.

By Proposition 3.4, we have $d_{T(\Delta)} \leq d_{F} \leq d_{T(R)}$ for every virtually isometric, holomorphic self-map $F: T(R) \rightarrow T(R)$.

We consider infinitesimal metrics of Teichmüller distances. Let $v$ be a tangent vector based at $p \in T(R)$ and $\alpha(t)$ a smooth curve on $T(R)$ with $\alpha(0)=p$ whose tangent vector at $t=0$ is $v$. Then the infinitesimal Teichmüller metric is obtained by

$$
\|v\|=\lim _{t \rightarrow 0} \frac{d_{T(R)}(\alpha(t), \alpha(0))}{t} .
$$

This is well-defined independently of the choice of $\alpha$. In fact, it is coincident with the Teichmüller norm (Finsler metric) on each tangent space of $T(R)$. Similarly, we have the infinitesimal universal Teichmüller metric $\|v\|_{T(\Delta)}$ restricted to $T(R)$.

The following claim says that these two metrics are globally biLipschitz equivalent. See [11, Theorem V.4.7].

Lemma 3.5. There exists a uniform constant $c>0$ such that

$$
c\|v\| \leq\|v\|_{T(\Delta)} \leq\|v\|
$$

for any tangent vector $v$ of $T(R)$.

For a holomorphic self-map $F$ of $T(R)$, we denote by $d F$ the derivative of $F$, which is a holomorphic self-map of the holomorphic tangent bundle $T(T(R))$ over $T(R)$. Note that the derivative and the iteration are commuting such as $d\left(F^{n}\right)=(d F)^{n}$.

Since $\left\|(d F)^{n}(v)\right\| \leq\|v\|$ by the non-expanding property, in parallel to Definition 3.3, we have a norm on each tangent space of $T(R)$ for which the derivative $d F$ is an isometry as

$$
\|v\|_{d F}:=\lim _{n \rightarrow \infty}\left\|(d F)^{n}(v)\right\| .
$$

This is the maximal metric dominated by the infinitesimal Teichmüller metric for which $d F$ is an isometry. On the other hand, we define the infinitesimal 
metric of $d_{F}$ as

$$
\delta_{F}(v)=\lim _{t \rightarrow 0} \frac{d_{F}(\alpha(t), \alpha(0))}{t},
$$

where $\alpha(t)$ is a smooth curve on $T(R)$ with $\alpha(0)=p$ whose tangent vector at $t=0$ is $v$.

Proposition 3.6. The infinitesimal metric $\delta_{F}(v)$ of $d_{F}$ is well-defined and coincident with $\|v\|_{d F}$.

Proof. By using a fact that $\lim _{t \rightarrow 0}$ exists uniformly on $n \in \mathbf{N}$ in the second line below, we have

$$
\begin{aligned}
\delta_{F}(v) & =\lim _{t \rightarrow 0} \frac{d_{F}(\alpha(t), \alpha(0))}{t} \\
& =\lim _{t \rightarrow 0} \lim _{n \rightarrow \infty} \frac{d_{T(R)}\left(F^{n}(\alpha(t)), F^{n}(\alpha(0))\right)}{t} \\
& =\lim _{n \rightarrow \infty}\left\|(d F)^{n}(v)\right\|=\|v\|_{d F} .
\end{aligned}
$$

Thus the assertions are verified.

By Proposition 3.6, we have $\|v\|_{T(\Delta)} \leq\|v\|_{d F} \leq\|v\|$ for a virtually isometric, holomorphic self-map $F: T(R) \rightarrow T(R)$ since $d_{T(\Delta)} \leq d_{F}$.

A key to the proof of Theorem 3.2 is to show that the $F$-invariant distance $d_{F}$ is biLipschitz equivalent to $d_{T(R)}$ in the following sense.

Lemma 3.7. For a virtually isometric, holomorphic self-map $F: T(R) \rightarrow$ $T(R)$, there exists a constant $c>0$ independent of $F$ such that

$$
c d_{T(R)}(p, q) \leq d_{F}(p, q) \leq d_{T(R)}(p, q)
$$

for any $p$ and $q$ in $T(R)$.

Proof. Since $d_{T(R)}$ and $d_{F}$ are inner distances, we have only to show that $c L_{d_{T(R)}}(\alpha) \leq L_{d_{F}}(\alpha)$ for any smooth curve $\alpha:[0,1] \rightarrow T(R)$. Here $L_{d_{T(R)}}(\alpha)=$ $\int_{0}^{1}\left\|\alpha^{\prime}(t)\right\| d t$ and $L_{d_{F}}(\alpha)=\int_{0}^{1}\left\|\alpha^{\prime}(t)\right\|_{d F} d t$ by Proposition 3.6. Since $c\|v\| \leq$ $\|v\|_{T(\Delta)} \leq\|v\|_{d F}$ by Lemma 3.5, we obtain the assertions.

Proof of Theorem 3.2. By Lemma 3.7, there is a constant $c>0$ such that $c d_{T(R)}(p, q) \leq d_{F}(p, q)$. Hence inequalities

$$
d_{F}(p, q)=d_{F}\left(F^{n}(p), F^{n}(q)\right) \leq d_{T(R)}\left(F^{n}(p), F^{n}(q)\right) \leq d_{T(R)}(p, q)
$$

yield the assertion. 


\section{Dynamics of GeOMetric SElF-EMBeddings OF TEICHMÜLleR SPACES}

In this section, we investigate dynamics of the geometric holomorphic selfembedding $f^{*}: T(R) \rightarrow T(R)$ induced by a holomorphic self-cover $f \in$ $\operatorname{Cov}(R)$, by applying the structure theorem discussed in Section 2 .

Since every holomorphic cover $f: R \rightarrow R^{\prime}$ of a Riemann surface $R$ onto another Riemann surface $R^{\prime}$ induces a holomorphic embedding $f^{*}: T\left(R^{\prime}\right) \rightarrow$ $T(R)$ between their Teichmüller spaces, the diagram of holomorphic covering in Theorem 2.1 yields the corresponding diagram as follows:

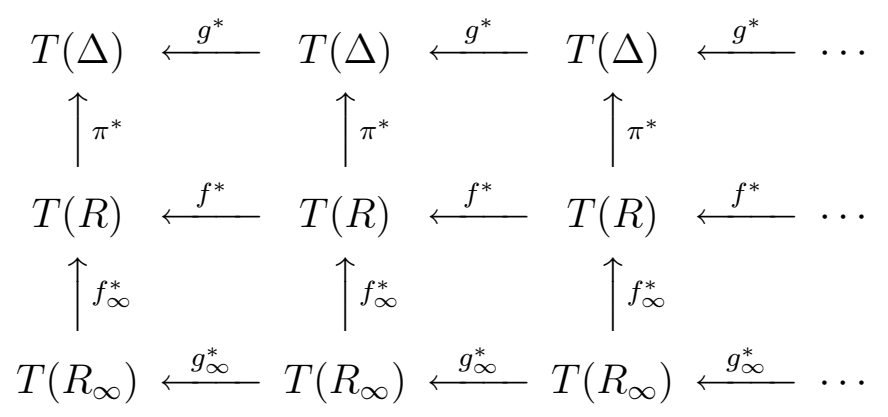

Recall that every holomorphic embedding in the diagram above preserves the base points. Moreover, a holomorphic cover of Riemann surfaces is noninjective if and only if the induced holomorphic embedding between Teichmüller spaces is non-surjective.

In general, let $R$ be a Riemann surface, $\pi: \Delta \rightarrow R$ a holomorphic universal cover, and $\Gamma$ the covering transformation group for $\pi$. The Bers embedding of the Teichmüller space $T(R)$ is well-defined for the holomorphic universal cover $\pi$, which we denote by $\beta_{\pi}: T(R) \rightarrow T_{B}(\Gamma)$. Let $R^{\prime}$ be another Riemann surface, $f: R \rightarrow R^{\prime}$ a holomorphic cover, and $\Gamma^{\prime}$ the covering transformation group for $f \circ \pi$. Let $f^{*}: T\left(R^{\prime}\right) \rightarrow T(R)$ be the holomorphic embedding induced by $f$. Then the Bers embedding $\beta_{f \circ \pi}: T\left(R^{\prime}\right) \rightarrow T_{B}\left(\Gamma^{\prime}\right)$ satisfies $\beta_{f \circ \pi}=\beta_{\pi} \circ f^{*}$. Therefore

$$
\beta_{\pi} \circ f^{*} \circ \beta_{f \circ \pi}^{-1}: T_{B}\left(\Gamma^{\prime}\right) \rightarrow T_{B}(\Gamma)
$$

is an inclusion map.

On the other hand, any conformal automorphism $g \in \operatorname{Aut}(\Delta)$ gives another universal cover $\pi \circ g: \Delta \rightarrow R$ and it defines the Bers embedding $\beta_{\pi \circ g}: T(R) \rightarrow$ $T_{B}\left(g^{-1} \Gamma g\right)$. Then this satisfies $\beta_{\pi \circ g}=g_{*} \circ \beta_{\pi}$, where

$$
g_{*}=\left(g^{-1}\right)^{*}: B(1) \rightarrow B(1)
$$

is the isometric linear automorphism defined by the push-forward of the holomorphic quadratic differentials in $B(1)$ by $g$.

We represent the diagram above in the Bers embedding. For the holomorphic universal covers $\pi$ and $f \circ \pi$ of $R$, we have two Bers embeddings of $T(R)$, which 
are $\beta_{\pi}: T(R) \rightarrow T_{B}(\Gamma)$ and $\beta_{f \circ \pi}: T(R) \rightarrow T_{B}\left(\Gamma_{1}\right)$. Then

$$
\beta_{\pi} \circ f^{*} \circ \beta_{f \circ \pi}^{-1}: T_{B}\left(\Gamma_{1}\right) \rightarrow T_{B}(\Gamma)
$$

is the inclusion map. On the other hand, when we represent $f^{*}$ as a self-map of $T_{B}(\Gamma)$, we consider the conjugation $\beta_{\pi} \circ f^{*} \circ \beta_{\pi}^{-1}$, which is coincident with $\beta_{f \circ \pi} \circ \beta_{\pi}^{-1}$. Since $f \circ \pi=\pi \circ g$, we have

$$
g_{*}=\beta_{\pi} \circ f^{*} \circ \beta_{\pi}^{-1}: T_{B}(\Gamma) \rightarrow T_{B}(\Gamma),
$$

whose image is $T_{B}\left(\Gamma_{1}\right)$. In a similar way, we have $\left(g_{*}\right)^{n}\left(T_{B}(\Gamma)\right)=T_{B}\left(\Gamma_{n}\right)$ for every $n \in \mathbf{N}$. Then it is easy to see the following claim.

Proposition 4.1. For a geometric self-embedding $f^{*} \in \operatorname{Cov}^{*}(T(R))$ and for any $n \in \mathbf{N}$, the image $\left(f^{*}\right)^{n}(T(R))$ of the $n$-th iteration of $f^{*}$ is a closed submanifold of $T(R)$ whose image under the Bers embedding $\beta_{\pi}: T(R) \rightarrow$ $T_{B}(\Gamma)$ is $T_{B}\left(\Gamma_{n}\right)$. Also the intersection $\bigcap_{n=1}^{\infty}\left(f^{*}\right)^{n}(T(R))$ is a closed subspace of $T(R)$ whose image under $\beta_{\pi}$ is $T_{B}\left(\Gamma_{\infty}\right)$.

In general, we define a characteristic subset of the dynamics of $F \in \operatorname{End}(T(R))$ as follows.

Definition 4.2. For a holomorphic self-map $F \in \operatorname{End}(T(R))$, we define the full cluster set of $F$ by $C(F)=\bigcap_{n=1}^{\infty} \overline{F^{n}(T(R))}$.

The full cluster set $C(F)$ is the maximal, closed, completely invariant set under the action of $F$. Proposition 4.1 can be paraphrased as follows.

Corollary 4.3. For a geometric self-embedding $f^{*} \in \operatorname{Cov}^{*}(T(R))$, the full cluster set $C\left(f^{*}\right)$ is identified with $T_{B}\left(\Gamma_{\infty}\right) \cong T\left(R_{\infty}\right)$, namely, it is coincident with $f_{\infty}^{*}\left(T\left(R_{\infty}\right)\right)$ in $T(R)$.

Remark 4.4. Similarly, the fixed point set $\operatorname{Fix}\left(f^{*}\right)$ of $f^{*} \in \operatorname{Cov}^{*}(T(R))$ is identified with $T_{B}(\hat{\Gamma}) \cong T(\hat{R})$. The Teichmüller space $T(\hat{R})$ is regarded as the quasiconformal deformation space of the complex dynamical system defined by the holomorphic self-cover $f: R \rightarrow R$. Namely, given $\hat{p} \in T(\hat{R})$, we have another holomorphic self-cover $f_{\hat{p}}: R_{p} \rightarrow R_{p}$ such that $f$ and $f_{\hat{p}}$ are quasiconformally conjugate by $R \rightarrow R_{p}$. For the complex dynamics induced by a rational map, this Teichmüller space has been studied in [16]. For instance, in Example 2.5, the Riemann surface $\hat{R}$ is the three-punctured sphere and hence $T(\hat{R})$ consists of a singleton.

We also give the definition of certain subsets concerning the dynamics of $F \in \operatorname{End}(T(R))$.

Definition 4.5. For a point $p \in T(R)$, it is said that $q \in T(R)$ is a $\omega$-limit point of $p$ for $F \in \operatorname{End}(T(R))$ if there exists a sequence $\left\{n_{i}\right\}$ of distinct positive integers such that $F^{n_{i}}(p)$ converge to $q$ as $i \rightarrow \infty$. The set of all $\omega$-limit points 
of $p$ for $F$ is called the $\omega$-limit set and is denoted by $\Lambda(F, p)$. It is said that $p \in T(R)$ is a recurrent point for $F$ if $p \in \Lambda(F, p)$. The set of all recurrent points for $F$ is called the recurrent set for $F$ and is denoted by $\operatorname{Rec}(F)$. The $\omega$-limit set for $F$ is defined by $\Lambda(F)=\bigcup_{p \in T(R)} \Lambda(F, p)$. Furthermore, $p \in T(R)$ is a periodic point for $F$ if there exists some $n \in \mathbf{N}$ such that $p \in \operatorname{Fix}\left(F^{n}\right)$. The set of all periodic points for $F$ is denoted by $\operatorname{Per}(F)$.

It is easy to see that the recurrent $\operatorname{set} \operatorname{Rec}(F)$ is a closed and completely invariant set under the action of $F$. Moreover, we have the following assertions.

Proposition 4.6. For a holomorphic self-map $F \in \operatorname{End}(T(R))$, the recurrent set $\operatorname{Rec}(F)$ is coincident with the limit set $\Lambda(F)$. It satisfies the inclusion relations

$$
C(F) \supset \operatorname{Rec}(F) \supset \overline{\operatorname{Per}(F)} \supset \operatorname{Fix}(F) .
$$

Proof. Since the inclusion relation $\operatorname{Rec}(F) \subset \Lambda(F)$ is clear by definition, we will show the other direction. For any $p \in \Lambda(F)$, there are some $q \in T(R)$ and an increasing sequence of positive integers $\left\{n_{i}\right\}$ such that $d_{T(R)}\left(F^{n_{i}}(q), p\right)$ converge to 0 as $i \rightarrow \infty$. Then the triangle inequality yields

$$
d_{T(R)}\left(F^{n_{i+1}-n_{i}}(p), p\right) \leq d_{T(R)}\left(F^{n_{i+1}-n_{i}}(p), F^{n_{i+1}}(q)\right)+d_{T(R)}\left(F^{n_{i+1}}(q), p\right) .
$$

Since $F^{n_{i+1}-n_{i}}$ is non-expanding, the first term in the right-hand side is not greater than $d_{T(R)}\left(p, F^{n_{i}}(q)\right)$. Hence we see that both terms in the right-hand side converge to 0 , which shows that $p \in \operatorname{Rec}(F)$.

The inclusion relation $\operatorname{Rec}(F) \subset C(F)$ is seen from the facts that $\operatorname{Rec}(F)$ is a closed, completely invariant set and that $C(F)$ is the maximal one among such sets. The other inclusions are obvious from the definition.

For a geometric self-embedding $f^{*} \in \operatorname{Cov}^{*}(T(R))$, we consider the restriction of $f^{*}$ to the full cluster set $C\left(f^{*}\right)$. This is equivalent to considering the biholomorphic automorphism $g_{\infty}^{*}$ of $T\left(R_{\infty}\right)$ induced by the conformal automorphism $g_{\infty} \in \operatorname{Conf}\left(R_{\infty}\right)$. In the Bers embedding, this is represented as the restriction of the linear isometry $g_{*}$ to $T_{B}\left(\Gamma_{\infty}\right)$. Then the next corollary comes from results in [4].

Corollary 4.7. A geometric self-embedding $f^{*} \in \operatorname{Cov}^{*}(T(R))$ satisfies the following: (1) The inclusion relations given in Proposition 4.6 for $F=f^{*}$ are proper; (2) For every point $p \in C\left(f^{*}\right)$, the orbit $O\left(f^{*}, p\right)=\left\{\left(f^{*}\right)^{n}(p) \mid n \in \mathbf{N}\right\}$ is nowhere dense in $C\left(f^{*}\right)$; (3) The set of periodic points $\operatorname{Per}\left(f^{*}\right)$ is nowhere dense in $C\left(f^{*}\right)$. 


\section{General Geometric Self-embeddings induced By QUASIREGULAR SELF-COVERS}

In this section, we investigate the dynamics of a geometric self-embedding $f^{*}: T(R) \rightarrow T(R)$ induced by a quasiregular self-cover $f \in \operatorname{QCov}(R)$. We assume that $f$ is non-injective in the arguments below, but the statements of the theorems are of course valid without this assumption.

Let $\pi: \Delta \rightarrow R$ a holomorphic universal cover and $\Gamma$ the covering transformation group for $\pi$. Take a lift $g: \Delta \rightarrow \Delta$ of $f$ such that $f \circ \pi=\pi \circ g$. This is a quasiconformal automorphism of $\Delta$ that conjugates $\Gamma$ to a Fuchsian group $\Gamma_{1}=g^{-1} \Gamma g$ containing $\Gamma$ properly. Then set $R_{1}=\Delta / \Gamma_{1}$ and let $f_{1}: R \rightarrow R_{1}$ be a holomorphic cover corresponding to the inclusion $\Gamma \varsubsetneqq \Gamma_{1}$. Since the universal cover $f_{1} \circ \pi: \Delta \rightarrow R_{1}$ has the covering transformation group $\Gamma_{1}$ that is conjugate to $\Gamma$ by $g$, there exists a quasiconformal automorphism $g_{1}: R_{1} \rightarrow R$ such that $g_{1} \circ f_{1} \circ \pi=\pi \circ g$. This in particular implies $g_{1} \circ f_{1}=f$, which gives the decomposition of the quasiregular cover $f$ into the quasiconformal homeomorphism $g_{1}$ and the holomorphic cover $f_{1}$. Note that this decomposition is uniquely determined up to conformal automorphisms of $R_{1}$. However, this ambiguity does not affect the global structure of the commutative diagram below.

In the same way, set $\Gamma_{2}=g^{-2} \Gamma g^{2}$ and $R_{2}=\Delta / \Gamma_{2}$. Then $\Gamma_{1} \varsubsetneqq \Gamma_{2}$ and this induces a holomorphic cover $f_{2}: R_{1} \rightarrow R_{2}$. Also the quasiconformal conjugation by $g$ induces a quasiconformal homeomorphism $g_{2}: R_{2} \rightarrow R_{1}$ such that $g_{2} \circ f_{2}=f_{1} \circ g_{1}$. Continuing this process, we have an increasing sequence of quasiconformally conjugate Fuchsian groups and their limit

$$
\Gamma \varsubsetneqq \Gamma_{1} \varsubsetneqq \Gamma_{2} \varsubsetneqq \cdots \subset \Gamma_{\infty}=\lim _{n \rightarrow \infty} \Gamma_{n}
$$

Finally, set $R_{\infty}=\Delta / \Gamma_{\infty}$. Since $g^{-1} \Gamma_{\infty} g=\Gamma_{\infty}$, there is a quasiconformal automorphism $g_{\infty}$ of $R_{\infty}$ induced by $g$. 
Summing up, we have the following diagram.

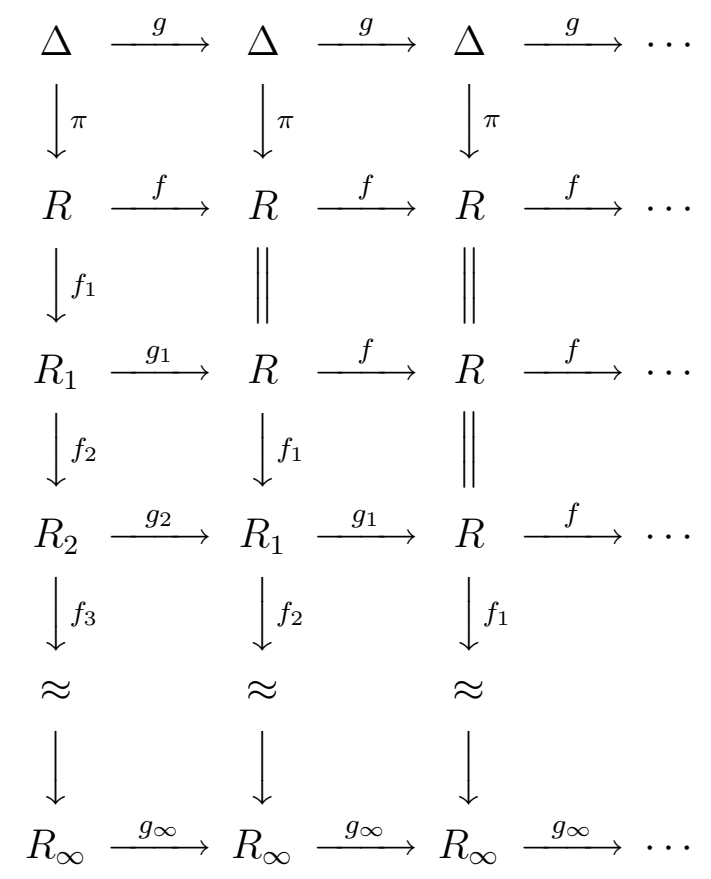

Here the vertical projection in each column from $R$ to $R_{\infty}$ is the holomorphic cover $f_{\infty}: R \rightarrow R_{\infty}$ satisfying $g_{\infty} \circ f_{\infty}=f_{\infty} \circ f$.

In general, a quasiregular cover $f: R \rightarrow R^{\prime}$ of a Riemann surface $R$ onto another Riemann surface $R^{\prime}$ induces a holomorphic embedding $f^{*}: T\left(R^{\prime}\right) \rightarrow$ $T(R)$ between their Teichmüller spaces, not necessarily preserving the base points. The diagram above induces a diagram between the corresponding Teichmüller spaces, where $T\left(R_{n}\right)(n=1,2, \ldots)$ are all biholomorphically equivalent to $T(R)$. The maps in the diagram consist of holomorphic embeddings $f^{*}: T(R) \rightarrow T(R)$ and $f_{n}^{*}: T(R) \rightarrow T(R)$, biholomorphic automorphisms $g_{n}^{*}: T(R) \rightarrow T(R)$ and a biholomorphic automorphism $g_{\infty}^{*}: T\left(R_{\infty}\right) \rightarrow T\left(R_{\infty}\right)$. In particular, we see that the image $\left(f^{*}\right)^{n}(o)$ of the base point of $T(R)$ under the $n$-th iteration of $f^{*}$ is $\left[\left(g_{1} \circ \cdots \circ g_{n}\right)^{-1}\right]$. Moreover, we have similar statements to Proposition 4.1 and Corollary 4.3 as follows.

Proposition 5.1. For a geometric self-embedding $f^{*} \in \operatorname{QCov}^{*}(T(R))$ and for any $n \in \mathbf{N}$, the image $\left(f^{*}\right)^{n}(T(R))$ is a closed submanifold of $T(R)$ whose image under the Bers embedding $\beta_{\pi}: T(R) \rightarrow T_{B}(\Gamma)$ is $T_{B}\left(\Gamma_{n}\right)$. Also the intersection $\bigcap_{n=1}^{\infty}\left(f^{*}\right)^{n}(T(R))$ is coincident with the full cluster set $C\left(f^{*}\right)$, which is a closed submanifold $f_{\infty}^{*}\left(T\left(R_{\infty}\right)\right)$ of $T(R)$ whose image under the Bers embedding $\beta_{\pi}$ is $T_{B}\left(\Gamma_{\infty}\right)$. 
Recall an invariant distance discussed in Section 3. Based on the consideration above, we give the following remark on invariant distances for geometric self-embeddings.

Remark 5.2. For a geometric self-embedding $f^{*} \in \operatorname{QCov}^{*}(T(R))$, we may take the universal Teichmüller distance $d_{T(\Delta)}$ as an $f^{*}$-invariant distance that meets condition $(* *)$. However, there is a possibly different invariant distance, which is the restriction of another Teichmüller distance on a larger space containing $T(R)$.

Let $g$ be the quasiconformal automorphism of $\Delta$ taken as above, which is the lift of $f \in \mathrm{QCov}(R)$. Define $\Gamma_{n}=g^{-n} \Gamma g^{n}$ for any negative integer $n<0$. Then a decreasing sequence of Fuchsian groups

$$
\Gamma \supset \Gamma_{-1} \supset \Gamma_{-2} \supset \cdots
$$

is given and the limit $\Gamma_{-\infty}=\bigcap_{n=1}^{\infty} \Gamma_{-n}$ is again a Fuchsian group. It satisfies $g^{-1} \Gamma_{-\infty} g=\Gamma_{-\infty}$. Consider the Riemann surface $R_{-\infty}=\Delta / \Gamma_{-\infty}$, which admits a quasiconformal automorphism $g_{-\infty}$ induced by $g$. Then $g_{-\infty}$ defines a biholomorphic automorphism $g_{-\infty}^{*}: T\left(R_{-\infty}\right) \rightarrow T\left(R_{-\infty}\right)$. Let $d_{T\left(R_{-\infty}\right)}$ denote the distance on $T(R)$ induced from the Teichmüller distance on $T\left(R_{-\infty}\right)$. Then $d_{T\left(R_{-\infty}\right)}$ is an $f^{*}$-invariant distance.

The Fuchsian group $\Gamma_{-\infty}$ may be trivial. In this case, $d_{T\left(R_{-\infty}\right)}=d_{T(\Delta)}$. In any case, the Bers embedding $T_{B}\left(\Gamma_{-\infty}\right)$ properly contains $\frac{\bigcup_{n=1}^{\infty} T_{B}\left(\Gamma_{-n}\right)}{\text {. }}$

Quasiregular self-covers of $R$ are quasiconformally conjugate to holomorphic self-covers of other Riemann surfaces $R^{\prime}$ in certain cases. In such cases, the geometric self-embeddings of $T(R)$ are essentially the same as those of $T\left(R^{\prime}\right)$ induced by the holomorphic self-covers. On the other hand, there is an example of a quasiregular self-cover that is not quasiconformally conjugate to any holomorphic self-cover. For instance, a quasiconformal automorphism $f$ of $R$ induced by the Dehn twist along a simple closed geodesic on $R$ is a kind of quasiregular self-cover, but it is not quasiconformally conjugate to any conformal automorphism. Thus, it is interesting to characterize the quasiregular self-covers that are realizable as holomorphic ones by quasiconformal conjugation, or more generally, by quasiconformal equivalence in the same Teichmüller class. Concerning this subject, our main theorem in this section is as follows.

Theorem 5.3. Let $f^{*} \in \mathrm{QCov}^{*}(T(R))$ be a geometric self-embedding induced by a quasiregular self-cover $f$ of a Riemann surface $R$. Then the following conditions are equivalent:

(1) there exist quasiconformal homeomorphisms $\phi: R \rightarrow R^{\prime}$ and $\phi^{\prime}: R \rightarrow$ $R^{\prime}$ in the same Teichmüller class $p_{0} \in T(R)$ such that $\phi^{\prime} \circ f \circ \phi^{-1}$ is a holomorphic self-cover of another Riemann surface $R^{\prime}$;

(2) $f^{*}$ has a fixed point $p_{0}$ in $T(R)$; 
(3) the set $\operatorname{Per}\left(f^{*}\right)$ of the periodic points of $f^{*}$ is not empty;

(4) the orbit $\left\{\left(f^{*}\right)^{n}(p)\right\}_{n \in \mathbf{N}}$ of any point $p \in T(R)$ is bounded.

Proof. $(1) \Rightarrow(2)$ : Let $f^{\prime}=\phi^{\prime} \circ f \circ \phi^{-1}$ be the holomorphic self-cover of $R^{\prime}$. The holomorphic embedding $f^{\prime *}: T\left(R^{\prime}\right) \rightarrow T\left(R^{\prime}\right)$ fixes the base point $o^{\prime}$ of $T\left(R^{\prime}\right)$. Since $\phi$ and $\phi^{\prime}$ induce the same biholomorphic homeomorphism $\phi^{*}: T\left(R^{\prime}\right) \rightarrow$ $T(R)$ satisfying $f^{*} \circ \phi^{*}=\phi^{*} \circ f^{\prime *}$, we see that $f^{*}$ fixes $\phi^{*}\left(o^{\prime}\right)=[\phi]=p_{0} \in T(R)$.

$(2) \Rightarrow(3) \Rightarrow(4)$ : Since $f^{*}$ is non-expanding, if the orbit of some point in $T(R)$ is bounded, then so is the orbit of every point in $T(R)$. The orbit of a periodic point is finite and in particular bounded. Thus proves the assertion.

$(4) \Rightarrow(1)$ : The biholomorphic automorphism $g_{\infty}^{*}: T\left(R_{\infty}\right) \rightarrow T\left(R_{\infty}\right)$ induced by the quasiconformal automorphism $g_{\infty}: R_{\infty} \rightarrow R_{\infty}$ satisfies $f^{*} \circ f_{\infty}^{*}=$ $f_{\infty}^{*} \circ g_{\infty}^{*}$. Since any orbit under $f^{*}$ is bounded in $T(R)$, any orbit under $g_{\infty}^{*}$ is also bounded in $T\left(R_{\infty}\right)$ since $f_{\infty}^{*}: T\left(R_{\infty}\right) \rightarrow T(R)$ preserves the infinity. Remark that, since $g_{\infty}^{*}$ is bijective, we can consider the backward orbit of $g_{\infty}^{*}$. It is easy to see that the forward orbit is bounded if and only if the backward orbit is bounded.

Then, by applying the next Theorem 5.4 to $g_{\infty}^{*}$, we see that $g_{\infty}^{*}$ has a fixed point in $T\left(R_{\infty}\right)$, which we denote by the Teichmüller class [ $\left.\phi_{\infty}\right]$ of a quasiconformal homeomorphism $\phi_{\infty}$ of $R_{\infty}$ onto another Riemann surface $R_{\infty}^{\prime}$. We can find another $\phi_{\infty}^{\prime}$ in the same Teichmüller class so that $g_{\infty}^{\prime}=\phi_{\infty}^{\prime} \circ g_{\infty} \circ \phi_{\infty}^{-1}$ is a conformal automorphism of $R_{\infty}^{\prime}$. Moreover, lifting $\phi_{\infty}$ and $\phi_{\infty}^{\prime}$ to $R$, we have quasiconformal homeomorphisms $\phi: R \rightarrow R^{\prime}, \phi^{\prime}: R \rightarrow R^{\prime}$ and a holomorphic cover $f_{\infty}^{\prime}: R^{\prime} \rightarrow R_{\infty}^{\prime}$ satisfying $\phi_{\infty} \circ f_{\infty}=f_{\infty}^{\prime} \circ \phi$ and $\phi_{\infty}^{\prime} \circ f_{\infty}=f_{\infty}^{\prime} \circ \phi^{\prime}$. Then we see that $f^{\prime}=\phi^{\prime} \circ f \circ \phi^{-1}$ is a holomorphic self-cover of $R^{\prime}$ because $f_{\infty}^{\prime} \circ f^{\prime}=g_{\infty}^{\prime} \circ f_{\infty}^{\prime}$ and $g_{\infty}^{\prime}$ is conformal.

Markovic [13] has proved that every uniformly quasisymmetric group is conjugate to a Fuchsian group by a quasisymmetric automorphism of the unit circle. This result generalizes the Nielsen realization problem (the fixed point problem on finite-dimensional Teichmüller spaces) to arbitrarily Teichmüller spaces and asserts that the bounded orbit of a quasiconformal mapping class subgroup yields a common fixed point on the Teichmüller space. More precisely, this in particular contains the following.

Theorem 5.4. Let $g_{\infty}: R_{\infty} \rightarrow R_{\infty}$ be a quasiconformal automorphism of a Riemann surface $R_{\infty}$ and $g_{\infty}^{*}: T\left(R_{\infty}\right) \rightarrow T\left(R_{\infty}\right)$ the biholomorphic automorphism of the Teichmüller space $T\left(R_{\infty}\right)$ induced by $g_{\infty}$. If the whole orbit $\left\{\left(g_{\infty}^{*}\right)^{n}(p)\right\}_{n \in \mathbf{Z}}$ of any point $p \in T\left(R_{\infty}\right)$ is bounded with respect to the Teichmüller distance, then $g_{\infty}^{*}$ has a fixed point in $T\left(R_{\infty}\right)$.

As a consequence of Theorem 5.3, we have an assertion finding a holomorphic self-cover under quasiconformal equivalence in the same Teichmüller class. 
First, we extend the structure theorem of self-covering of Riemann surfaces in Section 2 to the case where it is allowed to have branched points.

Lemma 5.5. Let $f: R \rightarrow R$ be a holomorphic branched self-cover of a Riemann surface $R$. Suppose that the grand orbit of the critical points of $f$ is discrete in $R$. Then there exist a holomorphic branched cover $f_{\infty}: R \rightarrow R_{\infty}$ and a conformal automorphism $g_{\infty}: R_{\infty} \rightarrow R_{\infty}$ of infinite order such that $g_{\infty} \circ f_{\infty}=f_{\infty} \circ f$.

Proof. Let $R^{\prime}$ be a Riemann surface obtained from $R$ by removing the grand orbit of the critical points of $f$. Then $f$ restricted $R^{\prime}$ is a holomorphic selfcover of $R^{\prime}$. Applying Theorem 2.1 to this $\left.f\right|_{R^{\prime}}$, we obtain a holomorphic cover $f_{\infty}^{\prime}: R^{\prime} \rightarrow R_{\infty}^{\prime}$ and a conformal automorphism $g_{\infty}^{\prime}: R_{\infty}^{\prime} \rightarrow R_{\infty}^{\prime}$ of infinite order. After that, we fill in all punctures of $R^{\prime}$ that come from the grand orbit of the critical points in order to recover $R$. By the condition that $g_{\infty}^{\prime} \circ f_{\infty}^{\prime}=\left.f_{\infty}^{\prime} \circ f\right|_{R^{\prime}}$, we see that the extension of $f_{\infty}^{\prime}$ by filling in the corresponding punctures of $R_{\infty}^{\prime}$ is well-defined and thus obtain a Riemann surface $R_{\infty}$ and a holomorphic branched cover $f_{\infty}: R \rightarrow R_{\infty}$. Also $g_{\infty}^{\prime}$ extends to a conformal automorphism $g_{\infty}: R_{\infty} \rightarrow R_{\infty}$.

Remark 5.6. Differently from Theorem 2.1, Lemma 5.5 does not state the construction of the Riemann surface $R_{\infty}$ explicitly. However, as in statement (7) of Theorem 2.1, the condition $g_{\infty} \circ f_{\infty}=f_{\infty} \circ f$ is able to characterize $R_{\infty}$ implicitly in terms of the highest cover among all such $f_{\infty}: R \rightarrow R_{\infty}$.

By Theorem 5.3 and Lemma 5.5, we have the following assertion concerning realization as holomorphic self-covering, which is related to a problem of complex dynamics of rational maps originating from Thurston (see [2], in particular, Remarks after Theorem 1).

Corollary 5.7. Let $f: R \rightarrow R$ be a quasiregular self-cover of a Riemann surface $R$ possibly branched. Suppose that the grand orbit $\hat{O}$ of the critical points of $f$ is discrete in $R$. Set $R_{0}=R-\hat{O}$ and consider the restriction of $f$ to $R_{0}$. If the orbit of any point $p \in T\left(R_{0}\right)$ under $f^{*}$, say $\left\{\left(f^{*}\right)^{n}(o)\right\}$, is bounded, then there exist quasiconformal homeomorphisms $\phi_{0}: R_{0} \rightarrow R_{0}^{\prime}$ and $\phi_{0}^{\prime}: R_{0} \rightarrow R_{0}^{\prime}$ in the same Teichmüller class of $T\left(R_{0}\right)$ such that they extend to $R$ quasiconformally and $\phi^{\prime} \circ f \circ \phi^{-1}$ is a holomorphic self-cover of another Riemann surface $R^{\prime}$.

In Theorem 5.3 and Corollary 5.7, we consider conditions for the quasiconformal equivalence $\phi^{\prime} \circ f \circ \phi^{-1}$ to be holomorphic. However, if we see the boundedeness of the maximal dilatation $K\left(f^{n}\right)$ of the $n$-th iteration of $f$ itself, we have a stronger conclusion that the quasiconformal conjugation $\phi \circ f \circ \phi^{-1}$ is holomorphic. Actually, this is an easier claim to obtain, for we do not have 
to rely on Theorem 5.4. Instead, we have only to use a technique of averaging the complex dilatations as in Tukia [20], where he has shown that any uniformly bounded quasiconformal group is quasiconformally conjugate to a Fuchsian group. Since the converse claim is also true, we may summarize them as follows.

Corollary 5.8. Let $f^{*} \in \mathrm{QCov}^{*}(T(R))$ be a geometric self-embedding induced by a quasiregular self-cover $f$ of a Riemann surface $R$. Then the following conditions are equivalent:

(1) there exist a quasiconformal homeomorphism $\phi: R \rightarrow R^{\prime}$ such that $\phi \circ f \circ \phi^{-1}$ is a holomorphic self-cover of another Riemann surface $R^{\prime}$;

(2) the maximal dilatation $K\left(f^{n}\right)$ of the $n$-th iteration of $f$ is uniformly bounded independently of $n \in \mathbf{N}$.

In Sullivan [19], there is an account of Corollary 5.7 (branched cover) in this conjugation version.

\section{Distribution of the isometric locus}

In Section 4, we have observed the dynamics of the geometric holomorphic self-embedding $f^{*} \in \operatorname{Cov}^{*}(T(R))$ by using its non-expanding property. In this section, we consider this property more closely.

The holomorphic self-embedding $f^{*}$ induces a holomorphic self-map

$$
\hat{f}^{*}: T(T(R)) \rightarrow T(T(R))
$$

of the holomorphic tangent bundle $T(T(R))$ over $T(R)$ such that $f^{*}$ maps $(p, v)$ to $\left(f^{*}(p), d f^{*}(v)\right)$, where $p$ is a point in $T(R)$ and $v$ is a tangent vector in the tangent space $T_{p}(T(R))$ at $p$. We define the magnification of a tangent vector $v \neq 0$ at $p$ by

$$
r_{f^{*}}(p, v):=\frac{\left\|d f^{*}(v)\right\|_{f^{*}(p)}}{\|v\|_{p}} .
$$

Here a tangent vector is measured by the Teichmüller norm $\|v\|_{p}$ defined on each tangent space $T_{p}(T(R))$. When there is no fear of confusion, we omit indicating $p$ and write $\|v\|$ instead of $\|v\|_{p}$.

If a covering $f: R \rightarrow R$ is amenable, then $r_{f^{*}}(p, v)=1$ for every $(p, v) \in$ $T(T(R))$. Namely, $f^{*}$ is an isometry with respect to the Teichmüller metric on $T(R)$. This has been proved in [14]. For example, finite covering and abelian covering are amenable. On the other hand, in this section, we will prove the following theorem for a non-amenable cover.

Theorem 6.1. If a holomorphic self-cover $f \in \operatorname{Cov}(R)$ is non-amenable, then the set

$$
D=\left\{(p, v) \in T(T(R)) \mid r_{f^{*}}(p, v)<1, v \neq 0\right\}
$$

is open and dense in the holomorphic tangent bundle $T(T(R))$. 
Note that it is trivial for $D$ to be open because $r_{f^{*}}$ is continuous. Theorem 6.1 follows from Theorem 6.4 due to McMullen [14] (see also [15]) combined with the fact that the Reich-Strebel functionals (tangent vectors) are dense in each tangent space $T_{p}(T(R))$.

Let $\operatorname{Belt}(R)$ be the Banach space of all Beltrami differentials $\mu$ on a Riemann surface $R$ with the norm $\|\mu\|_{\infty}=$ ess. sup $|\mu|$. Let $Q(R)$ be the Banach space of all integrable holomorphic quadratic differentials $\varphi$ on $R$ with the norm $\|\varphi\|_{1}=\int_{R}|\varphi|$. Then the tangent space $T_{o}(T(R))$ of $T(R)$ at the base point $o$ is identified with the dual space $Q(R)^{*}$. Each element $\mu \in \operatorname{Belt}(R)$ induces a bounded linear functional $v \in Q(R)^{*}$ by $v(\varphi)=\int \mu \varphi$. This gives a bounded linear operator $P: \operatorname{Belt}(R) \rightarrow Q(R)^{*}$ by $\mu \mapsto v=P(\mu)$. We say that $\mu \in$ $\operatorname{Belt}(R)$ represents $v \in Q(R)^{*}$ if $P(\mu)=v$.

The operator norm on $Q(R)^{*}$ is defined by

$$
\|v\|_{*}=\sup \left\{|v(\varphi)| \mid \varphi \in Q(R),\|\varphi\|_{1}=1\right\} .
$$

Since $Q(R)^{*}=T_{o}(T(R))$, this is also endowed with the Teichmüller norm

$$
\|v\|_{o}=\inf \left\{\|\mu\|_{\infty} \mid \mu \in \operatorname{Belt}(R), P(\mu)=v\right\} .
$$

It is clear that $\|v\|_{*} \leq\|v\|_{o}$. The inverse inequality follows from the HahnBanach theorem and the duality $\left(L^{1}\right)^{*}=L^{\infty}$. Hence $\|v\|_{*}=\|v\|_{o}$.

We say that $\mu \in \operatorname{Belt}(R)$ is extremal if $\|P(\mu)\|_{*}=\|\mu\|_{\infty}$. For each $v \in$ $Q(R)^{*}$, there exists an extremal $\mu \in \operatorname{Belt}(R)$ that represents $v$. This can be seen by $\|v\|_{*}=\|v\|_{o}$ and a fact that the infimum for $\|v\|_{o}$ is attained as the minimum.

The norm $\|\cdot\|_{p}$ on the tangent space $T_{p}(T(R))$ at each $p \in T(R)$ is defined in the same way as above by changing the base point of the Teichmüller space to $p$.

We say that $\left\{\varphi_{n}\right\} \subset Q(R)$ is a Hamilton sequence for $\mu \in \operatorname{Belt}(R)$ if $\left\|\varphi_{n}\right\|_{1} \leq$ 1 for all $n \in \mathbf{N}$ and

$$
\lim _{n \rightarrow \infty}\left|\int_{R} \mu \varphi_{n}\right|=\|\mu\|_{\infty}
$$

Moreover, it is a degenerating Hamilton sequence if, in addition, $\varphi_{n}$ converge compact uniformly to zero. We say that a tangent vector $v \in Q(R)^{*}=$ $T_{o}(T(R))$ is a Reich-Strebel functional if

$$
\inf \left\{\inf _{W}\left\|\left.\mu\right|_{R-W}\right\|_{\infty} \mid P(\mu)=v\right\}<\|v\|_{o},
$$

where $\inf _{W}$ is taken over all compact subsets $W$ of $R$.

The characterization of Reich-Strebel functionals is given as follows. This is an infinitesimal version of the characterization of Strebel points in the Teichmüller space. See [6, Section 4.8]. 
Proposition 6.2. An extremal Beltrami differential $\mu \in \operatorname{Belt}(R)$ represents a Reich-Strebel functional if and only if $\mu$ has no degenerating Hamilton sequence.

The following fact is useful, which can be also found in [6, Section 4.7].

Lemma 6.3. Suppose that $\mu \in \operatorname{Belt}(R)$ has no degenerating Hamilton sequence but has a Hamilton sequence $\left\{\varphi_{n}\right\}_{n \in \mathbf{N}}$ in $Q(R)$. Then there exist a subsequence $\left\{\varphi_{n(k)}\right\}_{k \in \mathbf{N}}$ of $\left\{\varphi_{n}\right\}$ and $\varphi \in Q(R)$ with $\|\varphi\|_{1}=1$ such that

$$
\lim _{k \rightarrow \infty}\left\|\varphi_{n(k)}-\varphi\right\|_{1}=0 .
$$

Proof. Since $\left\{\varphi_{n}\right\}$ is a normal family, we can choose a subsequence $\left\{\varphi_{n(k)}\right\}_{k \in \mathbf{N}}$ that converges uniformly on compact subsets. Moreover, the limit $\varphi$ belongs to $Q(R)$ and satisfies $\|\varphi\|_{1} \leq 1$. It is easy to see that the assumption $\left|\int_{R} \mu \varphi_{n}\right| \rightarrow$ $\|\mu\|_{\infty}$ implies that $\left\|\varphi_{n}\right\|_{1} \rightarrow 1$ as $n \rightarrow \infty$. Then, by [3, Lemma 7.2], we have

$$
\lim _{k \rightarrow \infty}\left\|\varphi_{n(k)}-\varphi\right\|_{1}=1-\|\varphi\|_{1} .
$$

Indeed, the triangle inequality gives

$$
0 \leq\left\|\varphi_{n}-\varphi\right\|_{1}+\|\varphi\|_{1}-\left\|\varphi_{n}\right\|_{1} \leq 2\|\varphi\|_{1},
$$

and then the dominated convergence theorem yields

$$
\lim _{k \rightarrow \infty}\left(\left\|\varphi_{n(k)}-\varphi\right\|_{1}+\|\varphi\|_{1}-\left\|\varphi_{n(k)}\right\|_{1}\right)=0 .
$$

To prove the assertion, we have only to show that $\|\varphi\|_{1}=1$. Suppose to the contrary that $\|\varphi\|_{1}<1$. Set

$$
\psi_{k}:=\frac{\varphi_{n(k)}-\varphi}{\left\|\varphi_{n(k)}-\varphi\right\|_{1}} .
$$

Then $\left\|\psi_{k}\right\|_{1}=1$ and $\left\{\psi_{k}\right\}_{k \in \mathbf{N}}$ converges to zero compact uniformly on $R$. On the other hand, since

$$
\left|\int_{R} \mu \psi_{k}\right| \geq \frac{\left|\int_{R} \mu \varphi_{n(k)}\right|}{\left\|\varphi_{n(k)}-\varphi\right\|_{1}}-\frac{\left|\int_{R} \mu \varphi\right|}{\left\|\varphi_{n(k)}-\varphi\right\|_{1}},
$$

we have

$$
\|\mu\|_{\infty} \geq \lim _{k \rightarrow \infty}\left|\int_{R} \mu \psi_{k}\right| \geq \frac{\|\mu\|_{\infty}}{1-\|\varphi\|_{1}}-\frac{\|\mu\|_{\infty}\|\varphi\|_{1}}{1-\|\varphi\|_{1}}=\|\mu\|_{\infty} .
$$

Hence $\lim _{k \rightarrow \infty}\left|\int_{R} \mu \psi_{k}\right|=\|\mu\|_{\infty}$, which means that $\left\{\psi_{k}\right\}_{k \in \mathbf{N}}$ is a Hamilton sequence for $\mu$. This contradicts the assumption that $\mu$ has no degenerating Hamilton sequence.

Let $f: S \rightarrow R$ be a holomorphic cover between Riemann surfaces $S$ and $R$. The pull-back of the Beltrami differentials is defined as usual and represented by the lift $\tilde{\mu} \in \operatorname{Belt}(S)$ of $\mu \in \operatorname{Belt}(R)$. On the other hand, the push-forward 
of the quadratic differentials is defined by the Poincaré series $\Theta(\phi) \in Q(R)$ for $\phi \in Q(S)$. The Poincaré series operator $\Theta: Q(S) \rightarrow Q(R)$ is surjective and the operator norm satisfies $\|\Theta\| \leq 1$. Moreover,

$$
\int_{R} \mu \Theta(\phi)=\int_{S} \tilde{\mu} \phi
$$

is satisfied for every $\mu \in \operatorname{Belt}(R)$ and every $\phi \in Q(S)$. The pull-back of the tangent vectors in $T_{o}(T(R))$ is also defined by the derivative $\left(d f^{*}\right)_{o}$ of the holomorphic embedding $f^{*}: T(R) \rightarrow T(S)$ at $o \in T(R)$. For every $\mu \in \operatorname{Belt}(R)$, we have $\left(d f^{*}\right)_{o}\left(P_{R}(\mu)\right)=P_{S}(\tilde{\mu})$, where $P_{R}: \operatorname{Belt}(R) \rightarrow Q(R)^{*}$ and $P_{S}: \operatorname{Belt}(S) \rightarrow Q(S)^{*}$. Therefore

$$
\left(d f^{*}\right)_{o}\left(P_{R}(\mu)\right)(\phi)=P_{S}(\tilde{\mu})(\phi)=P_{R}(\mu)(\Theta(\phi))
$$

for every $\phi \in Q(S)$.

A fundamental fact in our arguments is the following theorem proved by McMullen [14].

Theorem 6.4. Let $f: S \rightarrow R$ be a holomorphic cover of Riemann surfaces $S$ and $R$ and let $\Theta: Q(S) \rightarrow Q(R)$ be the Poincaré series operator induced by $f$. For every $\psi \in Q(R)-\{0\}$, we set

$$
N(\psi)=\sup \left\{\|\Theta(\phi)\|_{1} /\|\phi\|_{1} \mid \phi \in \Theta^{-1}(\langle\psi\rangle)\right\}(\leq 1),
$$

which is the operator norm of $\Theta$ restricted to the inverse image $\Theta^{-1}(\langle\psi\rangle)$ of the subspace spanned by $\psi$. Then $N$ is continuous on $Q(R)-\{0\}$. If $f$ is a non-amenable cover, then $N(\psi)<1$ for every $\psi \in Q(R)-\{0\}$.

A consequence from this theorem is as follows.

Theorem 6.5. Let $f: S \rightarrow R$ be a non-amenable holomorphic cover between Riemann surfaces $S$ and $R$ and let $\hat{f}^{*}: T(T(R)) \rightarrow T(T(S))$ be the holomorphic embedding between the holomorphic tangent bundles of the Teichmüller spaces induced by $f$. Assume that $v \in T_{p}(T(R))$ is a Reich-Strebel functional and $\mu \in \operatorname{Belt}\left(R_{p}\right)$ is an extremal differential that represents $v$. Let $\tilde{\mu} \in \operatorname{Belt}\left(S_{f^{*}(p)}\right)$ be the lift of $\mu$ to $S_{f^{*}(p)}$. Then the differential $\tilde{\mu}$ representing $d f^{*}(v)$ is not extremal. In particular, $\left\|d f^{*}(v)\right\|_{f^{*}(p)}<\|v\|_{p}$, that is, $r_{f^{*}}(p, v)<1$.

Proof. Suppose to the contrary that $\tilde{\mu}$ is extremal, that is,

$$
\sup _{\phi \in Q\left(S_{f^{*}(p)}\right),\|\phi\|_{1}=1}\left|\int_{S_{f^{*}(p)}} \tilde{\mu} \phi\right|=\|\tilde{\mu}\|_{\infty} .
$$

Then there is a sequence $\left\{\phi_{n}\right\}_{n \in \mathbf{N}} \subset Q\left(S_{f^{*}(p)}\right)$ such that $\left\|\phi_{n}\right\|_{1}=1$ and $\left|\int_{S_{f^{*}(p)}} \tilde{\mu} \phi_{n}\right| \rightarrow\|\tilde{\mu}\|_{\infty}$ as $n \rightarrow \infty$. Let $\Theta_{p}: Q\left(S_{f^{*}(p)}\right) \rightarrow Q\left(R_{p}\right)$ be the Poincaré series operator. Since $\left|\int_{S_{f^{*}(p)}} \tilde{\mu} \phi_{n}\right|=\left|\int_{R_{p}} \mu \Theta_{p}\left(\phi_{n}\right)\right|$, we see that 
$\left|\int_{R_{p}} \mu \Theta_{p}\left(\phi_{n}\right)\right| \rightarrow\|\mu\|_{\infty}$. Set $\psi_{n}:=\Theta_{p}\left(\phi_{n}\right) \in Q\left(R_{p}\right)$. It satisfies $\left\|\psi_{n}\right\|_{1} \leq$ $\left\|\phi_{n}\right\|_{1}=1$ by $\left\|\Theta_{p}\right\| \leq 1$. This means that $\left\{\psi_{n}\right\}_{n \in \mathbf{N}}$ is a Hamilton sequence for $\mu$. Moreover, $\left\|\psi_{n}\right\|_{1} \rightarrow 1$ as $n \rightarrow \infty$.

We apply Lemma 6.3 to $\mu \in \operatorname{Belt}\left(R_{p}\right)$ and $\left\{\psi_{n}\right\} \subset Q\left(R_{p}\right)$. Then there exist a subsequence of $\left\{\psi_{n}\right\}_{n \in \mathbf{N}}$, denoted by the same indices, and $\psi_{0} \in Q\left(R_{p}\right)$ such that $\psi_{n} \rightarrow \psi_{0}$. Next we apply Theorem 6.4 to see that $N\left(\psi_{n}\right) \rightarrow N\left(\psi_{0}\right)$ and $N\left(\psi_{0}\right)<1$. On the other hand, since

$$
N\left(\psi_{n}\right) \geq\left\|\Theta_{p}\left(\phi_{n}\right)\right\|_{1} /\left\|\phi_{n}\right\|_{1}=\left\|\psi_{n}\right\|_{1}
$$

and $\left\|\psi_{n}\right\|_{1} \rightarrow 1$, we have $N\left(\psi_{n}\right) \rightarrow 1$. This is a contradiction.

Now, for the proof of Theorem 6.1, we have only to remark the following fact. The proof is the same for the fact that the Strebel points are dense in $T(R)$. See [6, Section 4.11].

Proposition 6.6. For every $p \in T(R)$, the set of the Reich-Strebel functionals $v$ is dense in $T_{p}(T(R))$.

Theorem 6.1 says that generic tangent vectors in $T(T(R))$ are strictly contracted by $f^{*}$ if $f$ is non-amenable. However, we know that the magnification $r_{f^{*}}(p, v)$ is not uniformly bounded from above, for otherwise, the fixed point theorem says that the full cluster set $C\left(f^{*}\right)$ should consist of the unique fixed point of $f^{*}$; this is impossible by the fact that $C\left(f^{*}\right) \cong T\left(R_{\infty}\right)$ is not a singleton. We see more detailed information concerning this fact as follows.

Theorem 6.7. Let $f \in \operatorname{Cov}(R)$ be a non-amenable holomorphic cover. Then the following claims are satisfied.

(1) For every $(p, v) \in T(T(R))$ with $v \neq 0, \lim _{n \rightarrow \infty} r_{f^{*}}\left(\left(\hat{f}^{*}\right)^{n}(p, v)\right)=1$.

(2) For every $p \in \operatorname{Rec}\left(f^{*}\right)$, there exists a tangent vector $v \in T_{p}(T(R))$ with $v \neq 0$ such that $r_{f^{*}}(p, v)=1$.

Proof. (1) Suppose that $\liminf _{n \rightarrow \infty} r_{f^{*}}\left(\left(\hat{f}^{*}\right)^{n}(p, v)\right)<1$. Then, for some $\varepsilon>$ 0 , there exists an infinite sequence $\left\{m_{i}\right\}_{i \in \mathbf{N}}$ of positive integers such that $r_{f^{*}}\left(\left(\hat{f}^{*}\right)^{m_{i}}(p, v)\right)<1-\varepsilon$. However, for every $n \in \mathbf{N}$, we have

$$
r_{f^{*}}\left(\left(\hat{f}^{*}\right)^{n-1}(p, v)\right) \times \cdots \times r_{f^{*}}\left(\left(\hat{f}^{*}\right)(p, v)\right)=\frac{\left\|\left(d f^{*}\right)^{n}(v)\right\|_{\left(f^{*}\right)^{n}(p)}}{\|v\|_{p}} \geq c>0
$$

by the infinitesimal version of Theorem 3.2, which is seen by Lemma 3.5 and the fact that $\|v\|_{T(\Delta)}=\left\|\left(d f^{*}\right)^{n}(v)\right\|_{T(\Delta)}$. This is a contradiction.

(2) The Bers embedding $\beta_{\pi}: T(R) \rightarrow T_{B}(\Gamma)$ extends to their tangent bundles as $\hat{\beta}_{\pi}: T(T(R)) \rightarrow T\left(T_{B}(\Gamma)\right)$, where $T\left(T_{B}(\Gamma)\right)=T_{B}(\Gamma) \times B(\Gamma)$. Since the holomorphic embedding $f^{*}$ is represented by the linear isometry $g_{*}$ in the Bers embedding, we have

$$
\hat{g}_{*}:=\hat{\beta}_{\pi} \circ \hat{f}^{*} \circ \hat{\beta}_{\pi}^{-1}: T\left(T_{B}(\Gamma)\right) \rightarrow T\left(T_{B}(\Gamma)\right),
$$


where $\hat{g}_{*}(\varphi, \psi)=\left(g_{*}(\varphi), d g_{*}(\psi)\right)=\left(g_{*}(\varphi), g_{*}(\psi)\right)$ for every $(\varphi, \psi) \in T_{B}(\Gamma) \times$ $B(\Gamma)$.

Since $p \in \operatorname{Rec}\left(f^{*}\right)$, there exists an infinite sequence $\left\{n_{i}\right\}_{i \in \mathbf{N}} \subset \mathbf{N}$ such that $\left(f^{*}\right)^{n_{i}}(p) \rightarrow p$ as $i \rightarrow \infty$. For $\varphi_{p}=\beta_{\pi}(p)$, choose a tangent vector $(p, v) \in T(T(R))$ such that $\hat{\beta}_{\pi}(p, v)=\left(\varphi_{p}, \varphi_{p}\right)$. Then $\left(\hat{f}^{*}\right)^{n_{i}}(p, v)$ converge to $(p, v)$. By continuity of $r_{f^{*}}$, we have $r_{f^{*}}\left(\left(\hat{f}^{*}\right)^{n_{i}}(p, v)\right) \rightarrow r_{f^{*}}(p, v)$ as $i \rightarrow \infty$, and by the result of $(1)$, we see that $r_{f^{*}}(p, v)=1$.

In the remainder of this section, we investigate the above facts more closely on the tangent space $T_{o}(T(R))$ based at the origin $o \in T(R)$. As remarked before, we have the identification $T_{o}(T(R)) \cong\{0\} \times B(\Gamma)$ under the Bers embedding $\beta_{\pi}$. Then the derivative $d f^{*}: T_{o}(T(R)) \rightarrow T_{o}(T(R))$ turns to be the linear isometry $g_{*}: B(\Gamma) \rightarrow B(\Gamma)$ under this identification.

We define the following two sets:

$$
\begin{gathered}
I_{o}\left(d f^{*}\right)=\left\{v \in T_{o}(T(R)) \mid\left\|d f^{*}(v)\right\|=\|v\|\right\} ; \\
I_{o}^{\prime}\left(d f^{*}\right)=\left\{v \in T_{o}(T(R)) \mid\|v\|=\|v\|_{d f^{*}}\right\} .
\end{gathered}
$$

Both sets are closed and $I_{o}^{\prime}\left(d f^{*}\right)$ is forward invariant under $d f^{*}$. Moreover, we can define the recurrent set for $d f^{*}: T_{o}(T(R)) \rightarrow T_{o}(T(R))$ in the same way as before and denote it by $\operatorname{Rec}_{o}\left(d f^{*}\right)$.

Proposition 6.8. The inclusion relations $I_{o}\left(d f^{*}\right) \supset I_{o}^{\prime}\left(d f^{*}\right) \supset \operatorname{Rec}_{o}\left(d f^{*}\right)$ are satisfied.

Proof. Any tangent vector $v \in I_{o}^{\prime}\left(d f^{*}\right)$ satisfies $\|v\|_{d f^{*}}=\|v\|$ by definition. Since $\left\|d f^{*}(v)\right\|_{d f^{*}}=\|v\|_{d f^{*}}$ is valid for every $v \in T_{o}(T(R))$, we have

$$
\left\|d f^{*}(v)\right\|_{d f^{*}}=\|v\| \geq\left\|d f^{*}(v)\right\| .
$$

However, the inverse inequality is satisfied in general, and hence the above inequality turns to be equal. This shows the first inclusion $I_{o}\left(d f^{*}\right) \supset I_{o}^{\prime}\left(d f^{*}\right)$.

For the second inclusion, we first note that $I_{o}\left(d f^{*}\right) \supset \operatorname{Rec}_{o}\left(d f^{*}\right)$ holds by the same argument as in Theorem 6.7. Then, since $\operatorname{Rec}_{o}\left(d f^{*}\right)$ is completely invariant under $d f^{*}$, any tangent vector $v \in \operatorname{Rec}_{o}\left(d f^{*}\right)$ satisfies $\left\|\left(d f^{*}\right)^{n}(v)\right\|=$ $\|v\|$ for every $n \in \mathbf{N}$. This implies that $\|v\|_{d f^{*}}=\|v\|$ and hence $v$ belongs to $I_{o}^{\prime}\left(d f^{*}\right)$.

We have two kinds of partition of $T_{o}(T(R))$ by disjoint subsets: $\Lambda\left(d f^{*}\right):=$ $\operatorname{Rec}_{o}\left(d f^{*}\right)$ and its complement $\Omega\left(d f^{*}\right):=T_{o}(T(R))-\Lambda\left(d f^{*}\right) ; I_{o}\left(d f^{*}\right)$ and its complement $D_{o}\left(d f^{*}\right):=T_{o}(T(R))-I_{o}\left(d f^{*}\right)$. We have seen that $\Lambda\left(d f^{*}\right) \subset$ $I_{o}\left(d f^{*}\right)$ and we can see that $\Lambda\left(d f^{*}\right) \subset C\left(d f^{*}\right) \cong\{0\} \times B\left(\Gamma_{\infty}\right)$ in the same way as before. However, we have not known yet the answers to the following questions in the case where $f$ is non-amenable.

(1) Does $I_{o}\left(d f^{*}\right)$ include $\Lambda\left(d f^{*}\right)$ properly?

(2) Does $C\left(d f^{*}\right)$ contain $I_{o}\left(d f^{*}\right)$ ? 
(3) Does $C\left(d f^{*}\right)$ intersect $D_{o}\left(d f^{*}\right)$ ?

Conjecture 6.9. For a holomorphic non-amenable self-cover $f \in \operatorname{Cov}(R)$, the intersection $\Omega\left(d f^{*}\right) \cap I_{o}\left(d f^{*}\right)$ is a non-empty subset in $C\left(d f^{*}\right)$.

\section{REFERENCES}

[1] A. Beardon, Group of lifts of covering maps, unpublished manuscript (1989).

[2] A. Douady and J. Hubbard, A proof of Thurston's topological characterization of rational functions, Acta Math. 171 (1993), 263-297.

[3] C. Earle and F. Gardiner, Geometric isomorphisms between infinite dimensional Teichmüller spaces, Trans. Amer. Math. Soc. 348 (1996), 1163-1190.

[4] E. Fujikawa and K. Matsuzaki, Recurrent and periodic points for isometries of $L^{\infty}$ spaces, Indiana Univ. Math. J. 55 (2006), 975-997.

[5] F. Gardiner, Teichmüller Theory and Quadratic Differentials, John Wiley \& Sons, 1987.

[6] F. Gardiner and N. Lakic, Quasiconformal Teichmüller Theory, Math. Survey and Mono. 76, American Mathematical Society, 1999.

[7] Y. Imayoshi and M. Taniguchi, Introduction to Teichmüller Spaces, Springer-Tokyo 1992.

[8] T. Jørgensen, On discrete groups of Möbius transformations, Amer. J. Math. 98 (1976), 739-749.

[9] T. Jørgensen, A. Marden and C. Pommerenke, Two examples of covering surfaces, In: Riemann Surfaces and Related Topics, Ann. Math. Studies 97 (1978), 305-319.

[10] S. Kobayashi, Hyperbolic Complex Spaces, Springer, 1998.

[11] O. Lehto, Univalent Functions and Teichmüller Spaces, Graduate Text in Math. 109, Springer, 1986.

[12] V. Markovic, Biholomorphic maps between Teichmüller spaces, Duke Math. J. 120 (2003), 405-431 .

[13] V. Markovic, Quasisymmetric groups, J. Amer. Math. Soc. 19 (2006), 673-715.

[14] C. McMullen, Amenability, Poincaré series and quasiconformal maps, Invent. Math. 97 (1989), 95-127.

[15] C. McMullen, Amenable coverings of complex manifolds and holomorphic probability measures, Invent. Math. 110 (1992), 29-37.

[16] C. McMullen and D. Sullivan, Quasiconformal homeomorphisms and dynamics III. The Teichmüller space of a holomorphic dynamical system, Adv. Math. 135 (1998), 351-395.

[17] S. Morosawa, Y. Nishimura, M. Taniguchi and T. Ueda, Holomorphic Dynamics, Cambridge Univ. Press, 1999.

[18] S. Nag, The Complex Analytic Theory of Teichmüller Spaces, John Wiley \& Sons 1988.

[19] D. Sullivan, Conformal dynamical system, In: Geometric Dynamics, Lect. Notes Math. 1007, 725-752, Springer, 1983.

[20] P. Tukia, On two-dimensional quasiconformal groups, Ann. Acad. Sci. Fenn. 5 (1980), 73-78. 